Ambiances

anbiances Environnement sensible, architecture et espace urbain

$2 \mid 2016$

Ambiance et histoire de l'architecture : l'expérience et l'imaginaire sensibles de l'environnement construit

\title{
La restauration-conservation (2011-2019) de la Fondation Vasarely (1973-1976), ou comment rendre actuelles des ambiances surannées
}

Restoration-conservation (2011-2019) of the Foundation Vasarely (1973-1976), or how to return relevant of the sensory dimensions out of date?

Ana Bela de Araujo

\section{OpenEdition}

Journals

Édition électronique

URL : http://journals.openedition.org/ambiances/746

DOI : 10.4000/ambiances.746

ISSN : 2266-839X

Éditeur :

Direction Générale des Patrimoines - DAPA - MCC, UMR 1563 - Ambiances Architectures Urbanités (AAU)

Référence électronique

Ana Bela de Araujo, «La restauration-conservation (2011-2019) de la Fondation Vasarely (1973-1976), ou comment rendre actuelles des ambiances surannées ", Ambiances [En ligne], 2 | 2016, mis en ligne le 07 novembre 2016, consulté le 01 mai 2019. URL : http://journals.openedition.org/ambiances/746 ; DOI : 10.4000/ambiances.746

Ce document a été généré automatiquement le 1 mai 2019.

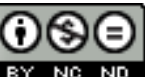

Ambiances is licensed under a Creative Commons Attribution-NonCommercial-NoDerivatives 4.0 International License. 


\section{La restauration-conservation (2011-2019) de la Fondation} Vasarely (1973-1976), ou comment rendre actuelles des ambiances surannées

Restoration-conservation (2011-2019) of the Foundation Vasarely (1973-1976), or how to return relevant of the sensory dimensions out of date?

\section{Ana Bela de Araujo}

L'auteur tient à remercier Pierre Vasarely, Claude Pradel-Lebar, Robert Jourdan, pour le temps qu'ils lui ont consacré pour des entretiens; Jacques Repiquet et Sylvie Denante pour l'ensemble des documents qu'ils ont mis à sa disposition et Sandrine Macke et Graziella Durio de la Fondation pour leur aide et soutien dans sa recherche documentaire.

Des façades à cercle d'argent, des chapelles rayonnantes comme les alvéoles d'une ruche, des parois se reflétant dans une pièce d'eau avec les ronds rouges du soleil en lueurs roses, dont on ne savait plus s'ils étaient vrais ou peints : c'était

l'inauguration du temple du cinétisme, le palais d'aluminium et de verre de la Fondation Vasarely. Sur chacune de ses 42 parois, des " intégrations » de céramique, de staff, de fer ou de verre peints, sur lesquelles se déversait une musique céleste composée à la fois de Bach et de jazz, savamment dosée. (L'œil de Vogue, mai 1976). 
1 La Fondation Vasarely (illustration 1) est un objet architectural singulier dont le caractère futuriste revendiqué dès l'origine en 1973 par son concepteur, le plasticien Victor Vasarely (1906-1997), a conservé sa puissance d'apostrophe. De loin, les façades métalliques aux plans pliés où les cercles inscrits dans des carrés jouent de l'alternance $\mathrm{du}$ noir et du blanc, créent une «œuvre-signe» à laquelle se suspend le regard (illustration 2). A l'intérieur, par contraste, c'est l'exaltation de la couleur à travers les 42 œuvres monumentales dont les jeux d'optique trompeurs déstabilisent le spectateur (illustration 3).

2 Le plan, régi par le principe combinatoire, est une juxtaposition de seize alvéoles hexagonales. Les sept qui sont destinées au parcours scénographique, ouvertes en leurs angles, désorientent le visiteur. Ce monument-écrin entièrement signé Vasarely, le contenu comme le contenant, a surpris parce qu'il ne ressemble à aucun autre. Machine à illusion, c'est une architecture manifeste de l'art optique de Vasarely, complètement lyrique mais rigoureusement construite. Là est son intérêt.

3 Le temps passe, mais mal sur cette œuvre d'art totale. Pas si mal en fait... Le temps a déposé sa patine, les couleurs sont passées, des désordres sont apparus. Mais aucune altération majeure n'est à déplorer. Un miracle que le monument doit à son histoire aux allures de saga. L'édifice a souffert du feuilleton judiciaire de la Fondation sur fond de finances englouties et de collection d'œuvres dilapidée ${ }^{1}$. Pendant ces décennies de lutte judiciaire, la priorité ne revient pas, de force, au bâtiment. A peine est-il entretenu. Une chance presque, car à l'aube du XXI ${ }^{\mathrm{e}}$ siècle, il est certes abîmé, un peu vieillot, mais « dans son jus ", authentique. Le folklore judiciaire a eu du bon.

Illustration 1 : Vue façade d'entrée de la Fondation Vasarely, Aix-en-Provence

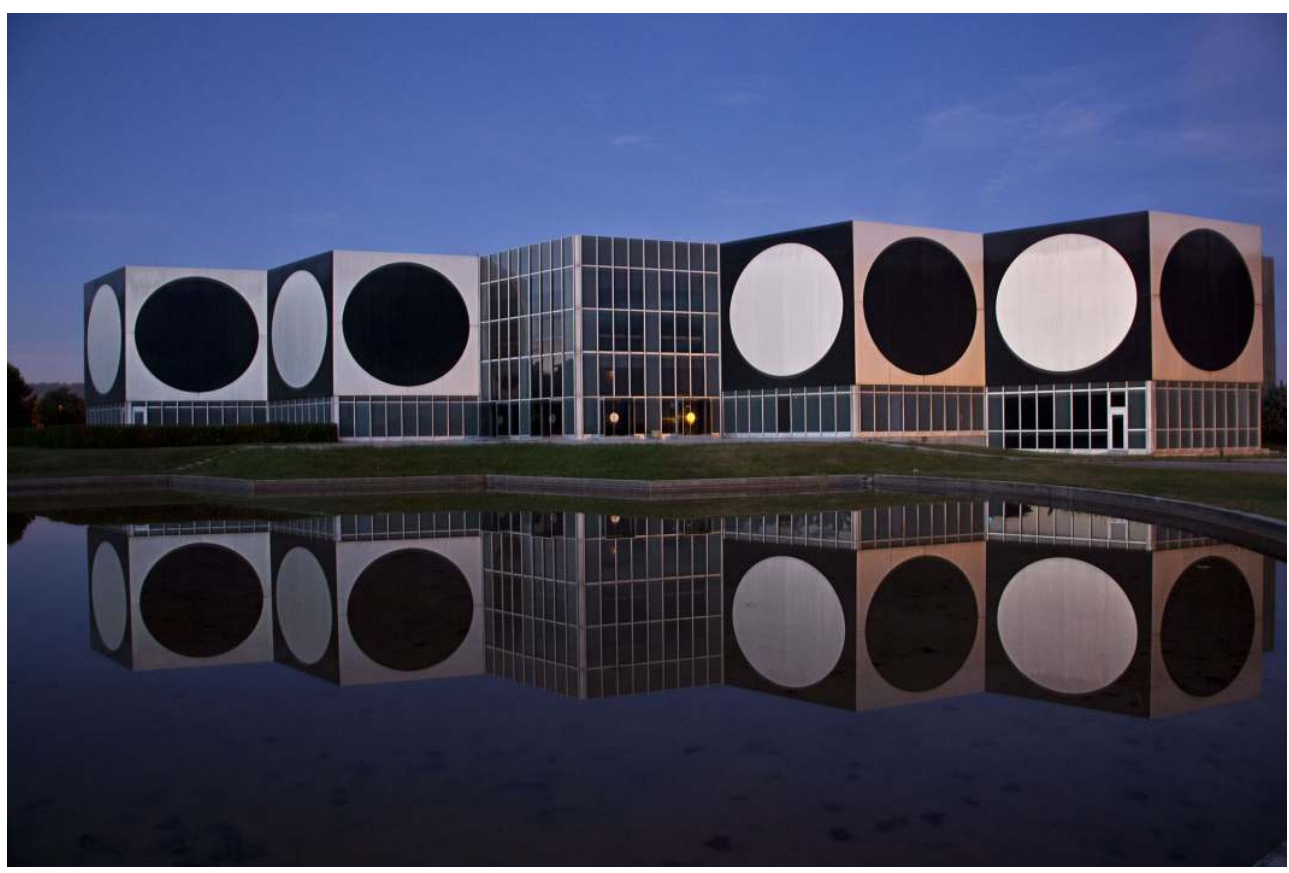

Source et copyright: Fondation Vasarely 
Illustration 2 : Salle 6, Fondation Vasarely. A gauche, Vega Anneaux, 1969-1975, 582x580 cm; au centre Tupa, 1972-1975, 562x506 cm ; à droite Ond, 1968-1975, 606x558 cm. Tapisseries réalisées à Aubusson

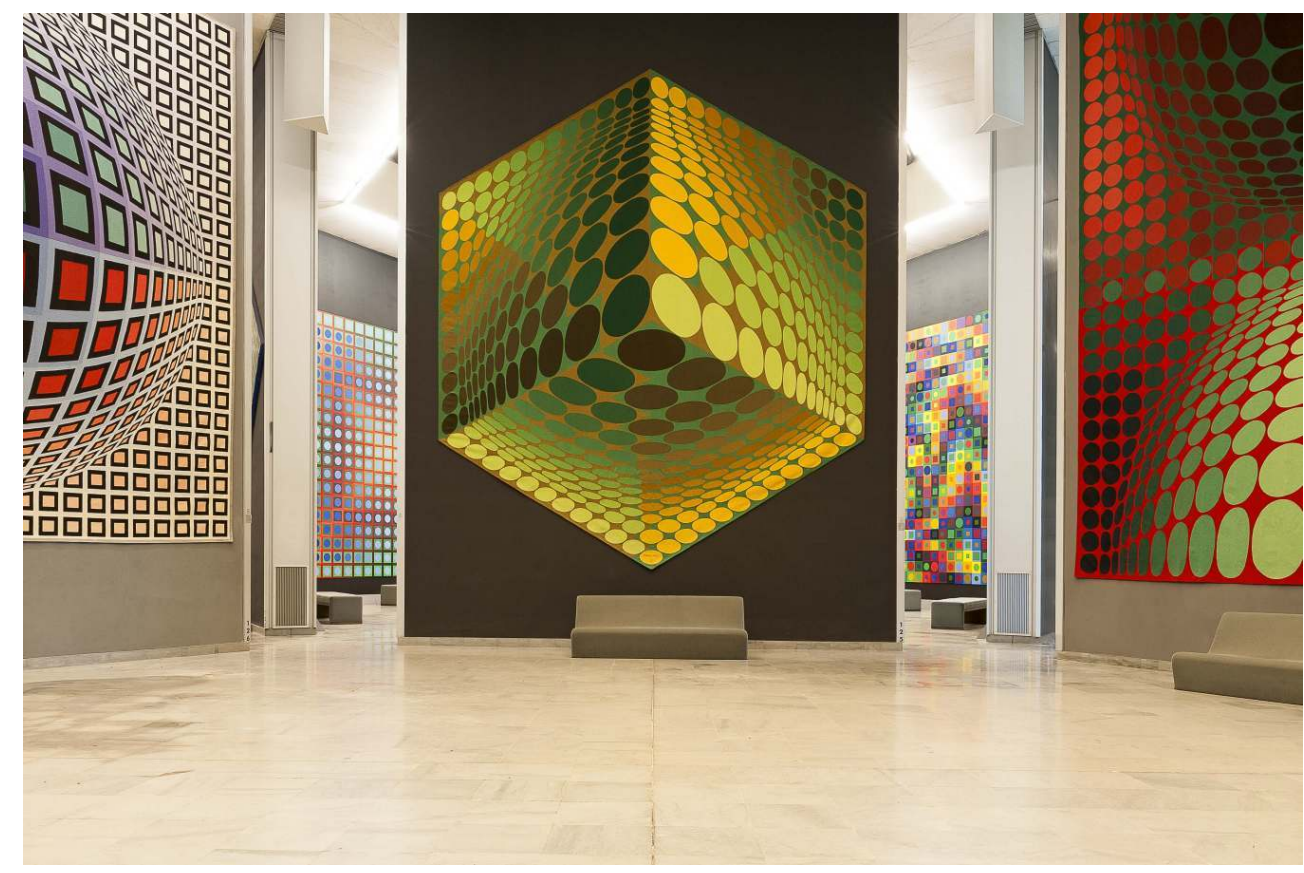

Source et copyright : Fondation Vasarely (c) Anne Fourès, Agence Luce

Illustration 3 : Plan masse de la Fondation Vasarely

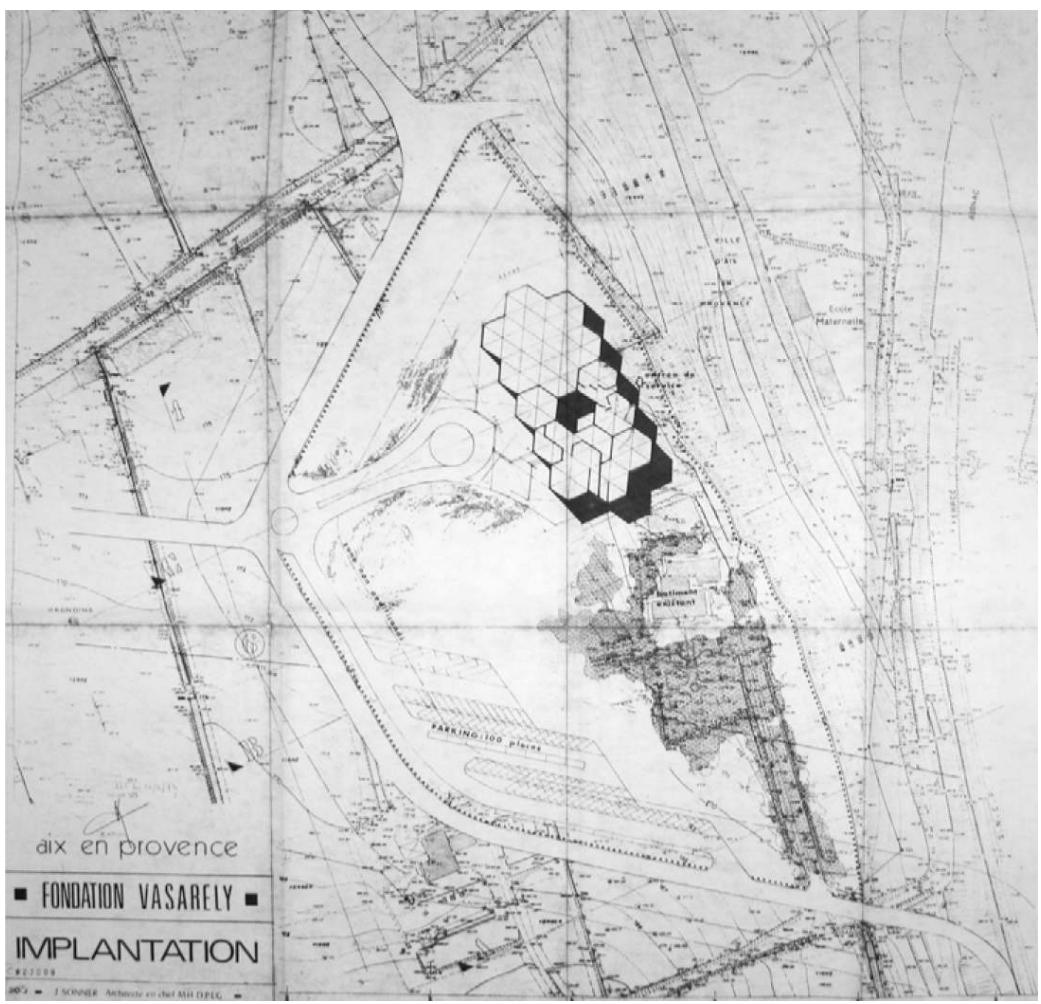

Source et copyright : Fondation Vasarely 
4 A telle enseigne que des marques de reconnaissance institutionnelle de l'intégrité et de l'originalité de l'édifice se succèdent. En 2001, un sursaut patrimonial sort de l'oubli cette œuvre significative des Trente Glorieuses : le bâtiment est labellisé Patrimoine du XX siècle. En 2003, il est inscrit à l'inventaire supplémentaire des monuments historiques. La renaissance du monument est en marche, confortée par la reprise en main de la Fondation par le petits-fils de l'artiste, Pierre Vasarely qui en devient le président en 2009. En 2010, la réhabilitation de l'édifice, au propre comme au figuré, est au programme de la prochaine décennie. Une équipe pluridisciplinaire d'architectes spécialistes du patrimoine moderne réalise une étude documentaire en vue de la réhabilitation de la Fondation. Leurs recherches historiques étayent le dossier de protection de la Fondation entamé par la Conservation régionale des monuments historiques. Le 14 janvier 2013, la commission nationale des Monuments Historiques classe à l'unanimité la Fondation. François Goven, Inspecteur général des Monuments historiques, précise alors que «cet édifice nous parvient aujourd'hui dans un état d'authenticité indéniable (contenu comme contenant), à la perception altérée, mais tout à fait "restaurable" ». ${ }^{2}$ L'heure n'est plus à la « réhabilitation » mais à la « restauration ».

5 Le parti patrimonial est fort, à l'opposé de toute démarche conciliatrice (Brandi, 1963) : on opte pour une restauration en conservation. L'authenticité de cet édifice, épargné par quarante ans de négligence en terme d'entretien, est désormais à respecter. L'enjeu est double: d'une part, sauvegarder l'édifice-écrin comme un témoignage historique, symbolisant le progrès industriel et technologique des Trente Glorieuses ; d'autre part, restaurer les œuvres exposées source de plaisir esthétique voire de fascination visuelle. Le dessein de cette restauration-conservation serait de révéler l'intégrité esthétique et historique de la Fondation, et par là-même l'intégrité "ambiantale » des espaces. Les dimensions constructives et techniques de l'édifice (parois en aluminium, double vitrage, pompes à chaleur...) étaient si expérimentales qu'elles inscrivent explicitement la Fondation dans le monde industrialisé des années 1970. De même, les effets scopiques et illusionnistes des damiers à renflements ou encore des moirages renvoient d'emblée les œuvres de Vasarely à cette atmosphère des années 1970. La restauration-conservation de l'ensemble serait-elle une projection volontaire dans une ambiance d'origine, donc datée voire surannée? Aujourd'hui le caractère désuet de l'espace incombe à la vétusté des textures, à l'écaillement des peintures, au laisser-aller général de deux décennies (pas de chauffage, pas de climatisation, pas de sécurisation). Rendre leur éclat à ces espaces est-ce leur rendre leur modernité déchue? Toute ambition de rétablir une «ambiance patrimoniale» n'est-elle pas une gageure? Toute tentative de patrimonialiser des qualités immatérielles, des sensations, "une atmosphère ", ne conduit-elle pas à créer une œuvre qui serait presque anachronique ? Notre propos est de questionner l'opération de restauration-conservation en cours de la Fondation Vasarely dans sa volonté et/ou capacité à recréer les espaces scénographiés d'origine souhaités par le plasticien. Interroger la dimension sensorielle de la restauration-conservation de la Fondation, c'est en somme se demander si les conduites perceptives et comportementales des visiteurs sont restaurables. Une restauration-conservation permet-elle à une expérience sensible passée de se réitérer de manière conforme dans le temps présent?

6 Dans un premier temps, nous nous attacherons à analyser la pensée du maître (illustration 4) - c'était son surnom - de ce lieu : sa conception de l'espace, la mise à contribution des architectes au service de son œuvre personnelle, les ambiances, la mémoire sensible à travers des photographies et des témoignages. Cette analyse sera 
ensuite mise en écho avec celle du projet actuel de restauration en conservation de la Fondation dans ses dimensions tant techniques que sensorielles.

Illustration 4 : Victor Vasarely dans l'alvéole 8 du Centre architectonique d'Aix-en-Provence, 1975

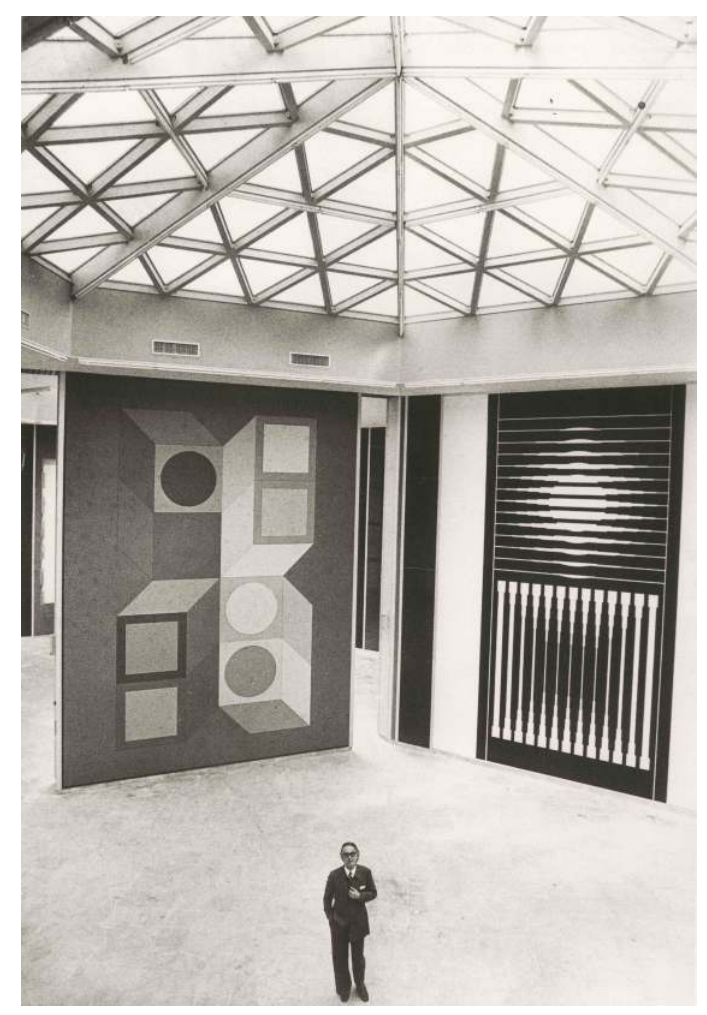

Source et copyright : Fondation Vasarely @ Anne Fourès, Agence Luce

\section{La Fondation de Victor Vasarely : une œuvre expérimentale singulière}

\section{Un programme artistique, architectural, social et culturel}

Dès 1966, Victor Vasarely, à 60 ans, pense à sa postérité. Il vient de participer à l'exposition "Responsive Eye » au Musée d'Art Moderne de New York, consacrée à l'Op Art (1965) qui a confirmé sa réputation internationale comme père de l'Art optique. Depuis plus de trente ans, il a inventé, par phases ${ }^{3}$, un langage abstrait universel compréhensible par tous en créant une nouvelle plastique cinétique. Il rêve à une fondation qui porterait son nom. Elle s'édifiera en deux temps, en deux lieux ${ }^{4}$. Le premier volet présente la partie la plus subjective de son œuvre, sorte de musée didactique. L'espace est inauguré en 1970, dans le château de Gordes, classé monument historique en 1931, entièrement restauré à ses frais (illustration 5). 
Illustration 5 : La Cité polychrome du bonheur. Etude sur carton, 1970, série « Intégrations murales »

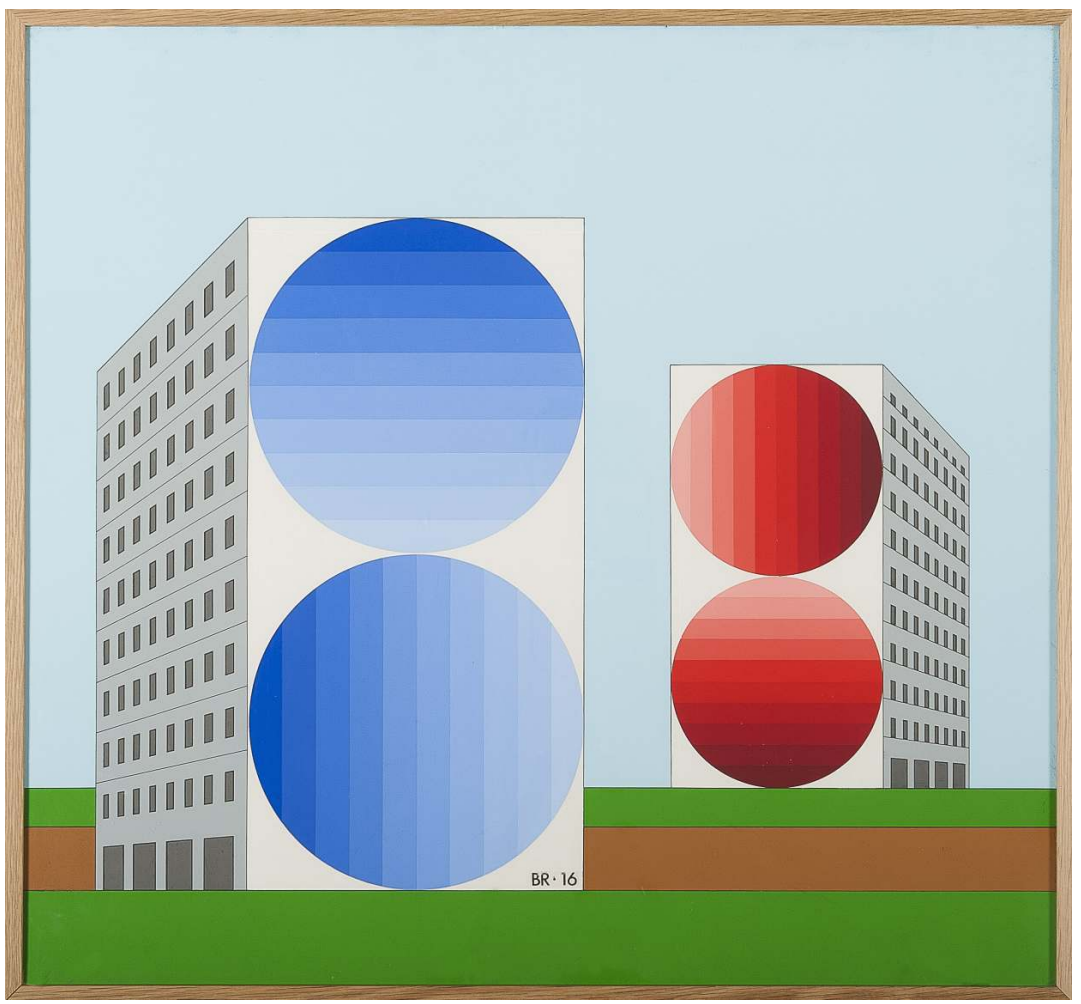

Source et copyright : Fondation Vasarely

A l'inverse, la seconde partie de la fondation doit incarner le projet architectural et social de l'artiste qui consiste à réaliser des œuvres plastiques monumentales et à les intégrer dans l'architecture et la ville. Il imagine un centre de recherche architectonique qui promeut l'intégration de la beauté plastique dans la cité, à l'image de la « cité polychrome du bonheur " qu'il défend afin de lutter contre les nuisances visuelles héritées du passé. Dans son esprit, la Fondation sera un lieu pour exposer son travail plastique au public, soit 44 intégrations monumentales mais aussi 798 recherches originales sur l'Art et la Cité. Vasarely l'imagine aussi lieu de rencontres et de recherches entre artistes, plasticiens, sculpteurs, architectes et urbanistes de tous pays.

Le choix de l'implantation est stratégique. En plein cœur d'un projet urbain de ZAC, le site est envisagé comme un terrain d'expérimentation optimal pour les intégrations. Surplombant un important réseau autoroutier, le terrain d'Aix-en-Provence près du Jasde-Bouffan offre aussi l'avantage d'assurer à l'édifice une visibilité idéale. En même temps, le terrain inscrit le projet dans une filiation picturale revendiquée par Vasarely: Cézanne, son "maître par excellence ", cet «initiateur génial des arts plastiques du présent $»^{5}$ selon les propres mots de Vasarely, avait vécu dans ce lieu baigné par la lumière du Midi, faisant face à la Montagne Sainte-Victoire. Dans ce lieu hautement symbolique, Vasarely décide pourtant de créer une fondation centrée sur elle-même, sans aucun lien visuel avec son environnement extérieur. La boîte est composée de façadestableaux animées par un effet d'optique basé sur un dynamisme rétinien du noir et du blanc. Visible depuis l'autoroute par des automobilistes circulant à $100 \mathrm{~km} / \mathrm{h}$, l'édifice s'inscrit pleinement dans une perception cinétique. Les piétons déambulant aux alentours 
de l'objet découvrent, eux, un effet de miroir, un objet posé sur une pelouse se reflétant dans un bassin.

En 1975, pour justifier son édifice futuriste Vasarely dira que "pour une institution d'avant-garde, il fallait construire un bâtiment ultramoderne ». Dès le début du projet, il a une idée très claire de son édifice. Depuis 1964, Vasarely est dans une nouvelle phase de réflexion artistique. S'intéressant à la structure cellulaire, il produit une série d'œuvres intitulée «L'hommage à l'hexagone ». Il base alors la conception esthétique de son édifice sur trois principes irréversibles: la trame hexagonale (des cellules hexagonales groupées), des façades aveugles et un éclairage zénithal. A charge de trouver des architectes pour traduire en plan, et exécuter son utopie artistique qu'il compte entièrement maîtriser. Un architecte parisien, Claude Pradel-Lebar, sera à ses côtés, en tant que conseiller en architecture, pour traduire en espace le concept cérébral du maitre. D'autres architectes auront la tâche de discipliner la volonté artistique par les contraintes techniques. Vasarely est pragmatique. Dans un pays aixois pétri d'histoire, le plus sûr pour que le projet ne rencontre aucune entrave est de faire appel précisément à l'architecte en chef des Monuments historiques de la région, Jean Sonnier ${ }^{6}$. Au service des envies de Vasarely, Sonnier assisté de son collaborateur Dominique Ronsseray, sera contraint d'expérimenter de nouvelles techniques et mises en œuvre.

\section{La géométrie pour le plan : la forme alvéolaire combinatoire ou comment agrandir son angle de vision}

11 Le concept de trame hexagonale est premier chez Vasarely, il est même antérieur au choix du site (illustration 6). Le bâtiment, unique, de 87 mètres de long par 40 de large, est constitué de seize alvéoles hexagonales accolées et hautes de 9 mètres. Sept d'entre elles, de toute hauteur, sont dédiées à la présentation des Intégrations (illustration 7). Ces œuvres monumentales occupent chaque paroi des hexagones, dont les angles à $120^{\circ}$ offrent un angle de vision aisé pour chacune d'entre elles. Chacune des parois sera ainsi perçue comme une entité autonome. 
Illustration 6 : Plan du rez-de-chaussée de la Fondation Vasarely

EMMLACEMENT PKISES ELECIKIGUES

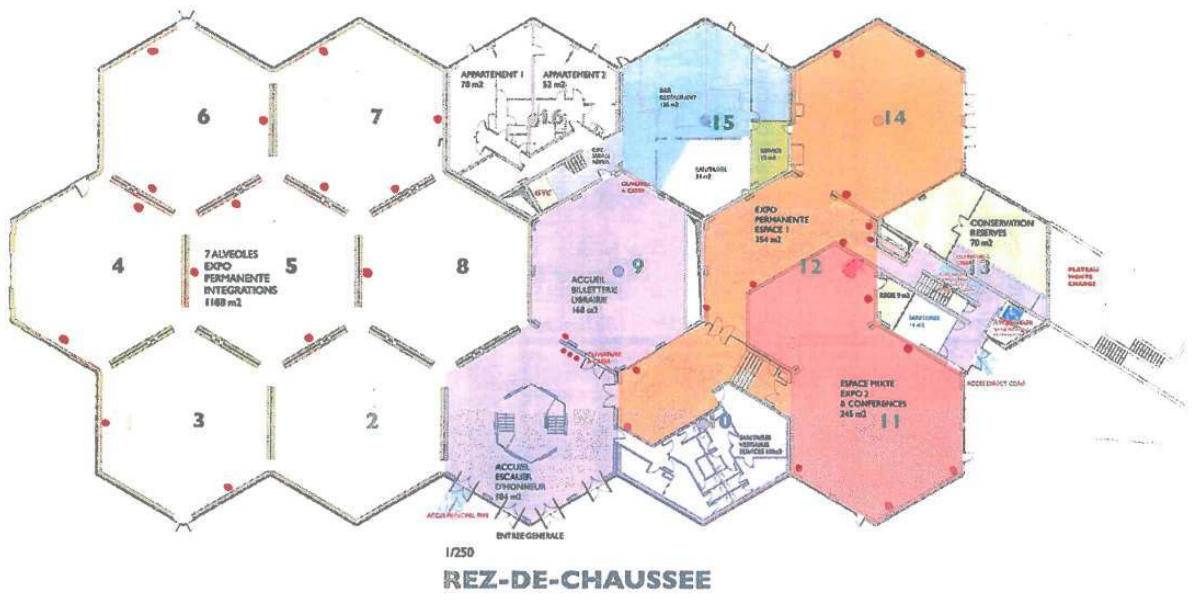

Source et copyright : Fondation Vasarely

Illustration 7 : A gauche, intégration Zett, 1966-1975, 528x528cm ; au centre Madjus, 1964-1975, $576 \times 576 \mathrm{~cm}$; à droite Kezdi, 1966-1975, 570x570 cm

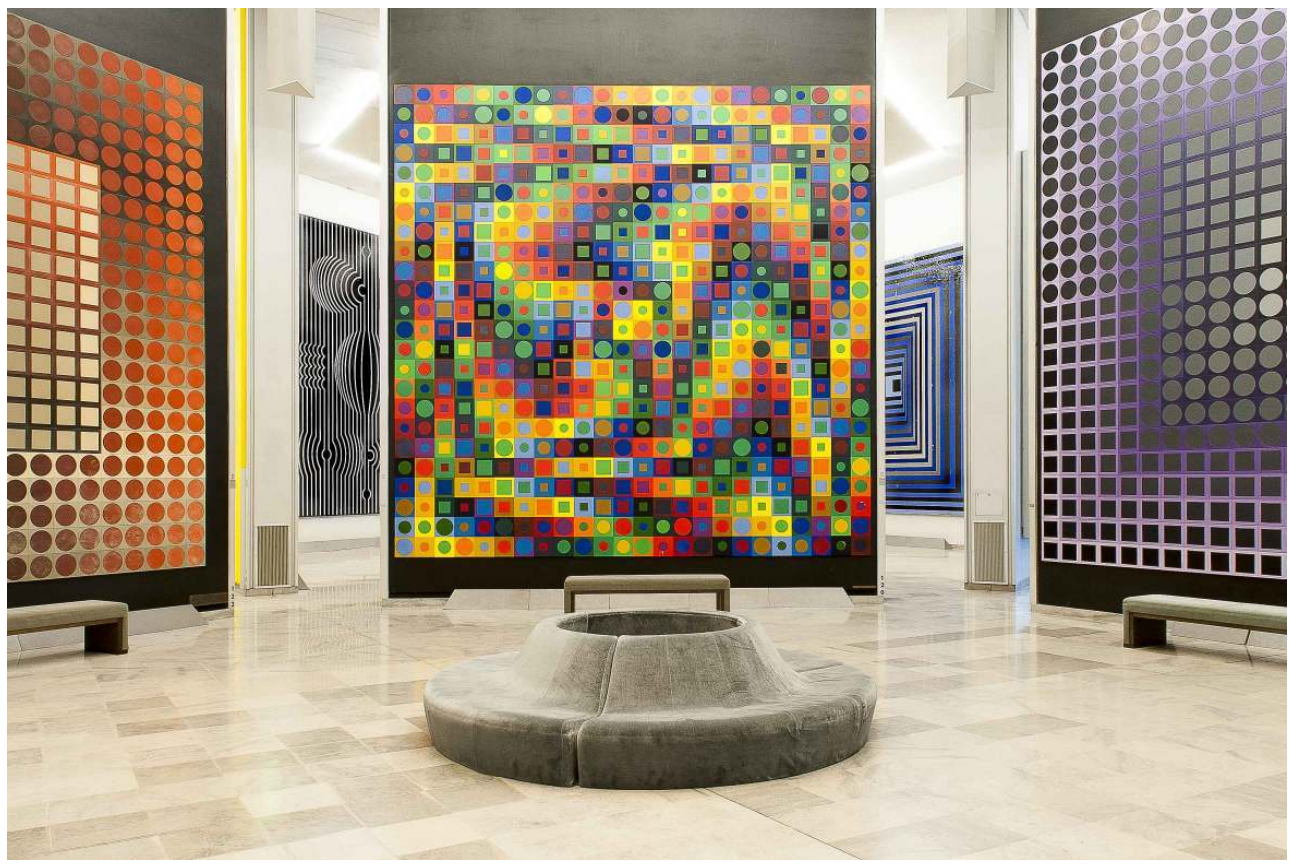

Source et copyright : Fondation Vasarely (c) Anne Fourès, Agence Luce

12 Ouvertes aux extrémités de chacune de leur face, les sept pièces hexagonales communiquent entre elles, laissant au visiteur le choix de son parcours. Les autres alvéoles sont divisées en deux niveaux, - excepté une d'entre elles destinée à un patio -, 
et sont réservées à un centre de recherche, une salle de conférence, une salle d'exposition, la bibliothèque, l'administration et l'accueil. L'ensemble est construit en éléments de béton préfabriqués et modulaires, les murs intégrant circuits et gaines.

Si la Fondation est un sanctuaire de la géométrie, elle est aussi le repaire de la couleur. Dans sa recherche d'un langage plastique universel, Vasarely avait mis au point dès 1948 un vocabulaire normalisé, son alphabet plastique, basé sur des unités plastiques permutables (combinaison de formes simples) qui, conjuguées à des gammes de couleur, permettent des combinatoires aux possibilités infinies. Pour ce faire, Vasarely avait créé des couleurs. Partant des six couleurs de base, un jaune de chrome, un vert émeraude, un outremer, un violet de cobalt, un rouge ainsi qu'un noir et un blanc, il a composé une gamme de vingt nuances, allant du ton très clair $n^{\circ} 6$ au ton très foncé $n^{\circ} 14$ (illustration 8).

Illustration 8 : Salle d'exposition au $1^{\text {er }}$ étage

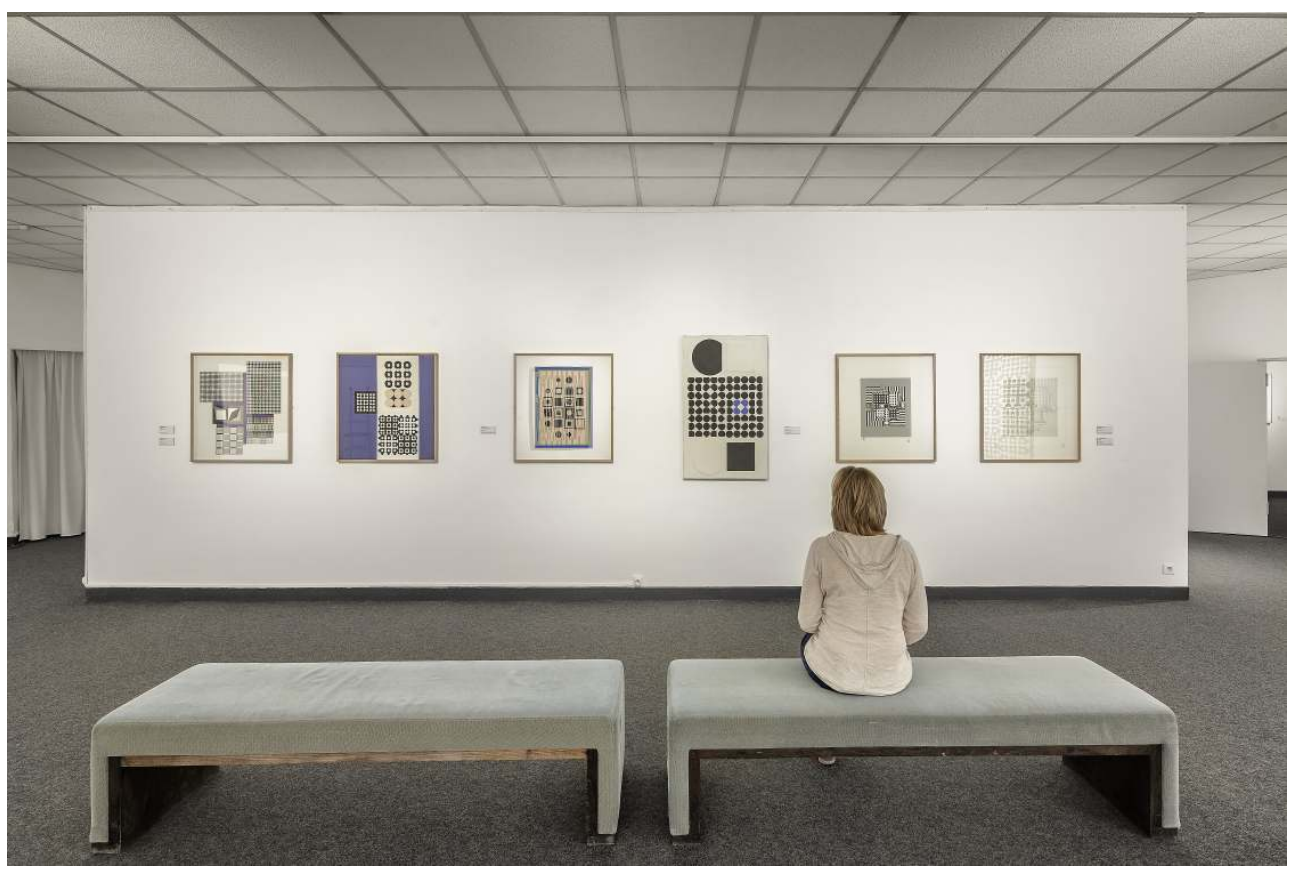

Source et copyright : Fondation Vasarely @ Aldo Amoretti

A Aix, pour l'atmosphère générale, il a choisi le ton $n^{\circ} 7$ autour du blanc et du noir. Une ambiance en camaïeu de gris, ce gris que Vasarely jugeait indispensable à l'ambiance studieuse de son atelier d'Annet-sur-Marne. La palette de couleurs des peintures murales s'étale du blanc au blanc cassé, à l'argenté, aux gris n² 2, 4, 6, 7 (Agence Gatier, 2011, p. 30) ; les éléments de serrurerie sont en aluminium anodisé ; les revêtements de sols (tapis, dalflex ou moquette) sont tous gris, quoique différents, et la pierre Calladaire, pierre marbrière grise des Alpes, choisie pour les espaces de prestige, accentue l'ambiance neutre de l'intérieur (illustration 9). Même le mobilier des salles d'expositions et de l'auditorium conçu par Emile Veranneman participe de cette atmosphère monocorde. S'ajoute l'aspect lisse, poli, sans aucune aspérité de toutes les textures, qui produit un environnement « glissant ", sourd et tiède. 


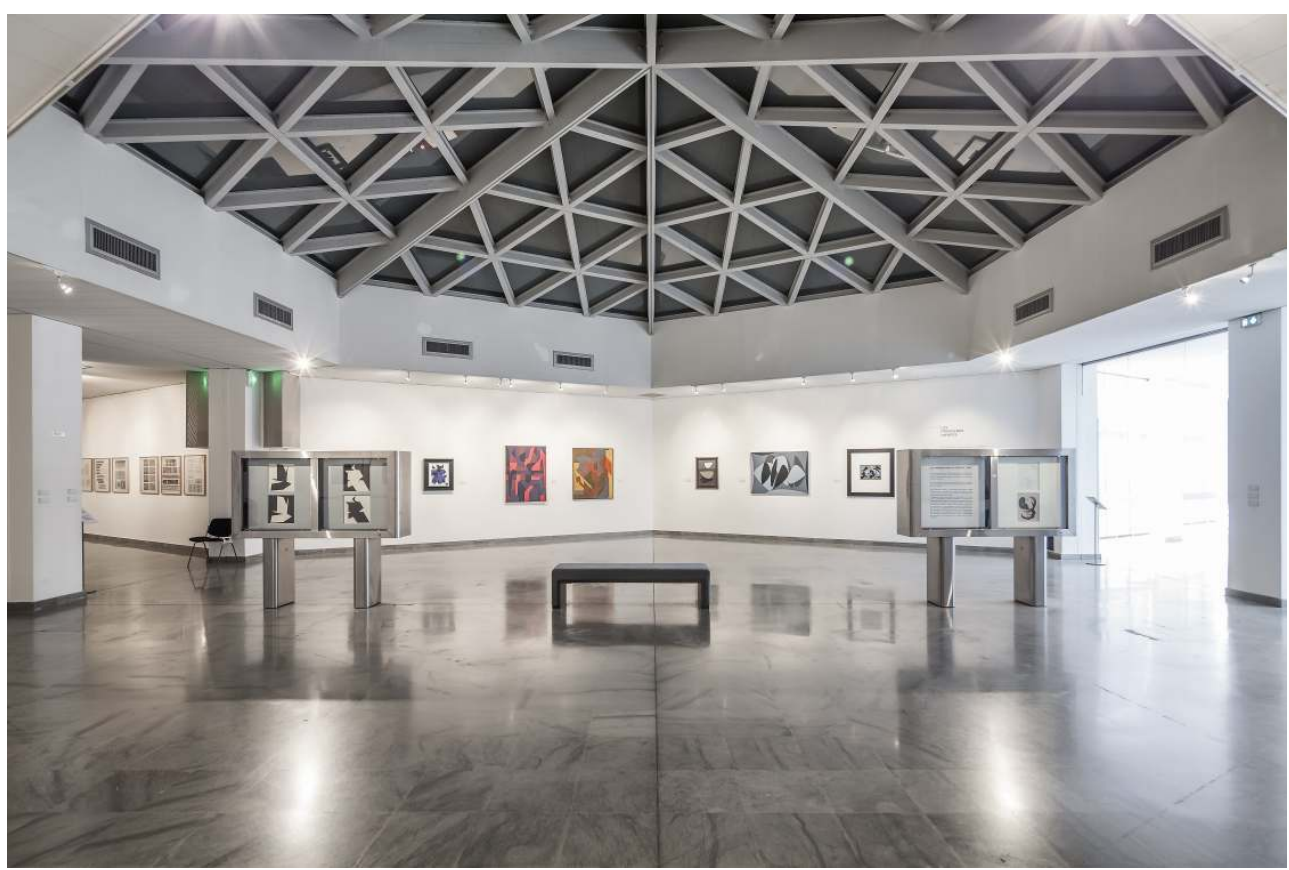

Source et copyright : Fondation Vasarely (c Aldo Amoretti

\section{Façades aveugles pour un monde clos ou comment ne regarder que soi}

Pour Vasarely qui se dit peu touché par le paysage sur le plan esthétique (Vasarely, 1979a, p. 74), il n'y a aucun inconvénient à ce que sa Fondation soit hermétique à son environnement. De ces façades aveugles, il en fait même des façades-tableaux reposant sur un rythme binaire de noir et de blanc, souvenir de son goût pour les séries d'unités formelles, comme l'échiquier avec ces cases régulières alternées, qui le fascine depuis l'enfance (ibid.a p. 38). Vingt-et-un panneaux décorés constituent la partie haute des façades, la partie basse du rez-de-chaussée étant vitrée par des murs-rideaux. Douze tôles d'aluminium devaient être assemblées pour composer chacun des panneaux en donnant l'illusion d'une seule tôle carrée de plus de $8 \mathrm{~m}$ de côté. Le recours à des techniques de pointe s'est avéré nécessaire pour réaliser ces façades géométriques pulsatives aux joints invisibles (illustration 10). 


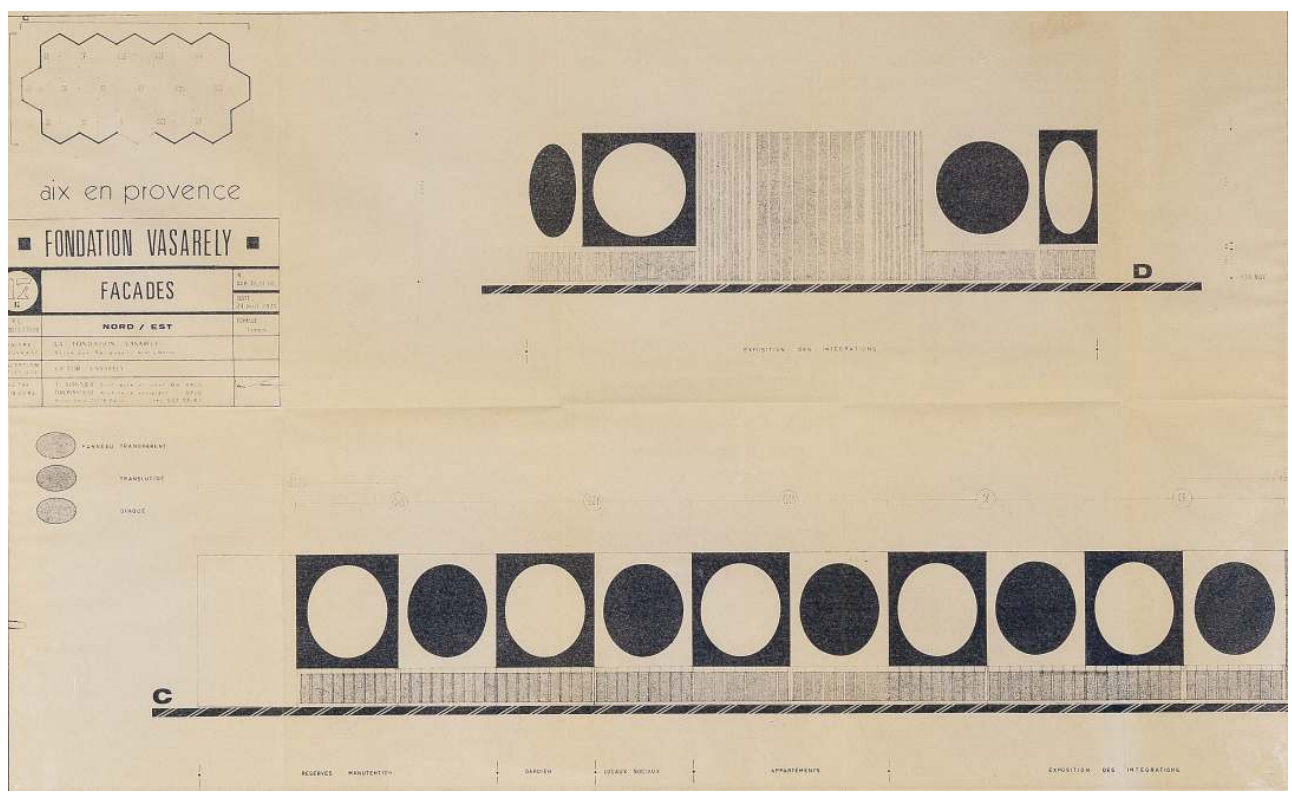

Source et copyright : Fondation Vasarely @ Anne Fourès, Agence Luce

16 Les difficultés d'usinage et de mise en œuvre se succèdent : une seule usine en Europe possède un train de laminoirs pouvant traiter les formats des plaques $(2,20 \mathrm{~m} \times 7,30 \mathrm{~m}$ par $6 \mathrm{~mm}$ d'épaisseur), un seul anodiseur en France a des cuves assez grandes, le problème d'homogénéité des teintes, la difficulté de la dilatation des plaques à cause de la chaleur et leur planéité (Sonnier, s.d.). Derrière la pureté affichée de cet édifice ultramoderne se cache un haut degré de technicité. Loin des compétences habituelles des architectes des monuments historiques, ce projet techniquement sophistiqué s'est construit par tâtonnements expérimentaux. L'aptitude des architectes, mais aussi des entreprises des monuments historiques à œuvrer au cas par cas, dans le détail et de manière artisanale, a permis de dompter la complexité propre à la mise en œuvre d'un idéal. Mais pour Vasarely, « la satisfaction de voir réaliser un vieux rêve n'a pas de prix » (Vasarely, 1979a, p. 214). Il sera très fier du résultat :

Cet ensemble s'impose dans le site par la pureté de sa découpe et l'apparente simplicité de sa géométrie. Le blanc et le noir mat de la façade en aluminium mettent curieusement en valeur la gamme de vert olivâtre du paysage cher à Cézanne que l'on découvre en approchant du bâtiment. Cette monochromie extérieure n'est pas neutre, car en même temps qu'elle assure une parfaite intégration au site, elle ménage au visiteur qui pénètre dans l'édifice la surprise $d u$ monde coloré qui l'attend. (Vasarely, 1979b)

\section{Un éclairage zénithal ou comment être dedans comme dehors}

Réaliser un bâtiment aveugle suppose un éclairage artificiel... ou alors une lumière zénithale. Victor Vasarely a opté pour la lumière du ciel, celle qui, dans un espace clos, lui a procuré son premier choc esthétique alors qu'il était enfant :

En fait c'est dans un endroit inattendu, au Városliget, un parc public de Budapest, que j'ai éprouvé la plus grande émotion esthétique de ma jeunesse. On trouvait dans ce parc un pavillon en forme de rotonde, dont l'intérieur était orné d'une fresque circulaire d'Arpád Feszti, racontant l'entrée du conquérant Arpád en Hongrie à la 
tête des Magyars [sic] $]^{7}$. La lumière diffusée par le haut, donnait une incroyable sensation: j'avais l'impression d'être devant un fragment de réalité, et non pas devant un panorama peint, comme si je me trouvais au milieu d'un champ, en plein soleil. J'étais ébloui par ce trompe-l'œil d'une échelle hors du commun. (Vasarely, 1979a, p. 36)

Pour obtenir la même qualité de lumière à l'intérieur qu'à l'extérieur, quatorze des prismes hexagonaux sont couverts par des coupoles translucides en verre (illustrations 11 et 12). Chaque coupole est une structure pyramidale pointant vers le ciel, réalisée par une maille triangulaire en bois lamellé-collé peinte en gris, qui supporte 96 composants verriers. Le complexe de toiture élaboré propose un vitrage supérieur en verre securit, un vitrage inférieur en verre triplex isolant et entre les deux une résille alvéolaire d'aluminium formant un brise-soleil. Pour contrecarrer l'effet de serre des dômes translucides, un important système de climatisation original a été élaboré. L'installation toute électrique repose sur un procédé optimisé d'économie d'énergie, fonctionnant sur la récupération de chaleur avec une circulation en double circuit établie pour les parties publiques dans l'épaisseur des murs, et des boîtes de mélange placées dans les fauxplafonds (Sonnier, s.d.). Dans une ambiance lumineuse et tempérée, le visiteur n'est pas distrait par l'extérieur, il est entièrement concentré par les œuvres monumentales colorées. Tel était le dessein de Vasarely.

Illustration 11 : Construction de la Fondation Vasarely, vers 1975

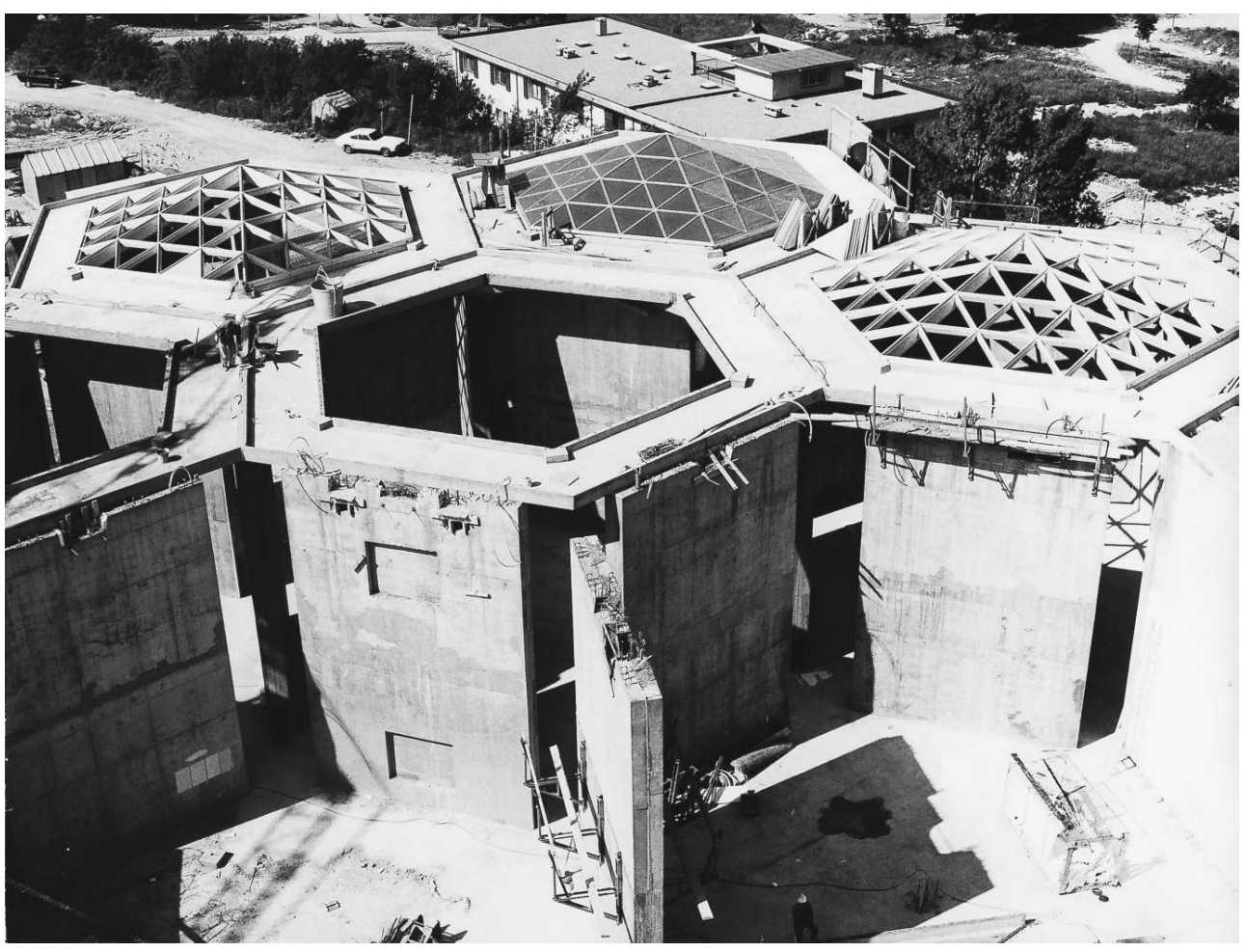

Source et copyright : Fondation Vasarely 
Illustration 12 : Victor Vasarely dans le bâtiment du Centre architectonique en construction, 1974

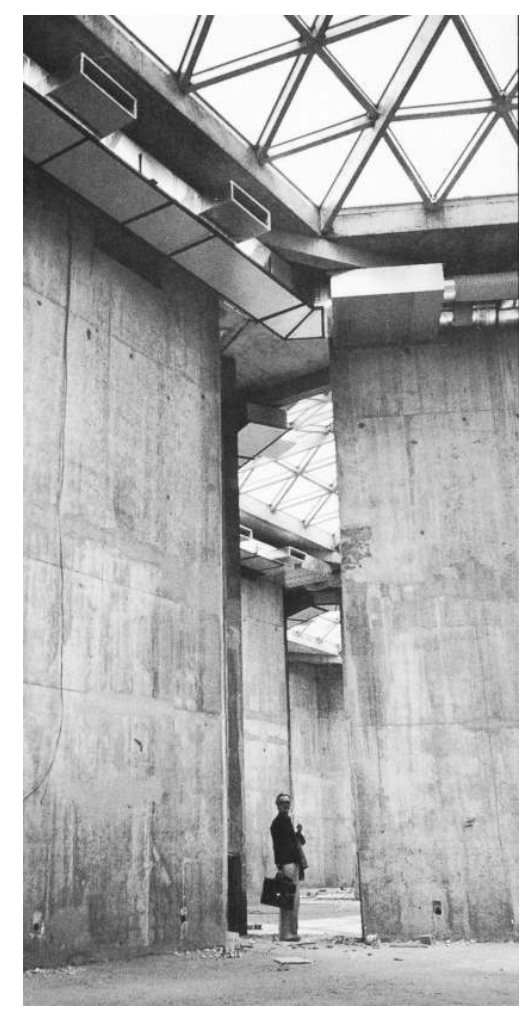

Source et copyright : Fondation Vasarely @ A Anne Fourès, Agence Luce

\section{La réception critique des ambiances}

19 A cette architecture de murs, sans ouvertures sur l'extérieur, répond à l'intérieur une explosion de couleurs à travers les 42 Intégrations de Vasarely. "Surprise : la couleur éclate, fuse, se balade, tourbillonne, mariée au noir, au blanc, au bleu du ciel. Fantasmagorie, délire, provocation?", s'interroge une journaliste (Demoriane, 1976, p. 88). C'est la gaieté contre la grisaille de nos villes, aurait pu lui rétorquer Vasarely (illustration 13).

Qualifiée d'art optique, son œuvre joue avec la perception visuelle. Juxtapositions de couleurs contrastées, enchaînements de dessins géométriques, illusions de mouvement, ambiguïtés spatiales, autant de dispositifs artistiques qui font du spectateur un œil réceptif ${ }^{8}$ et un corps actif. L'artiste précise : « le spectateur étant soumis à des variations perceptives, l'œuvre ne prend tout son sens que dans une certaine durée, au cours de laquelle elle lui paraît changer » (Vasarely, 1979a, p. 182).

21 A l'intérieur, les 42 œuvres sollicitent la participation du spectateur. Le visiteur progresse dans la découverte d'ambiances colorées, de jeux d'optique, de transparence, de reflet qui le projettent dans un univers instable, mouvant, labyrinthique qu'il doit interpréter. Il est vite étourdi, perdu, en totale insécurité.

Sa perception est constamment mise à mal, comme devant les déformations de l'œuvre Vega Anneaux, sorte de gonflements qui proposent des formes en plan mais qui donnent l'illusion d'un spectaculaire volume (illustrations 14 et 15). Vasarely est un magicien et sa fondation une machine à illusions. Vasarely s'en explique : 
Pour moi, le cinétisme est ce qui se passe dans l'esprit du spectateur quand son œil est obligé d'organiser un champ perceptif tel qu'il est nécessairement instable. Autrement dit, la réalité qui se présente à lui n'est pas une réalité donnée, qui serait la «bonne » vision de l'œuvre ; il y a au contraire plusieurs réalités qui alternent, selon les mécanismes qui relèvent strictement de la physiologie. (Vasarely, 1979a, p. 184).

Illustration 13 : Au fond Centauri, intégration composée de trois glaces, 1965-1975
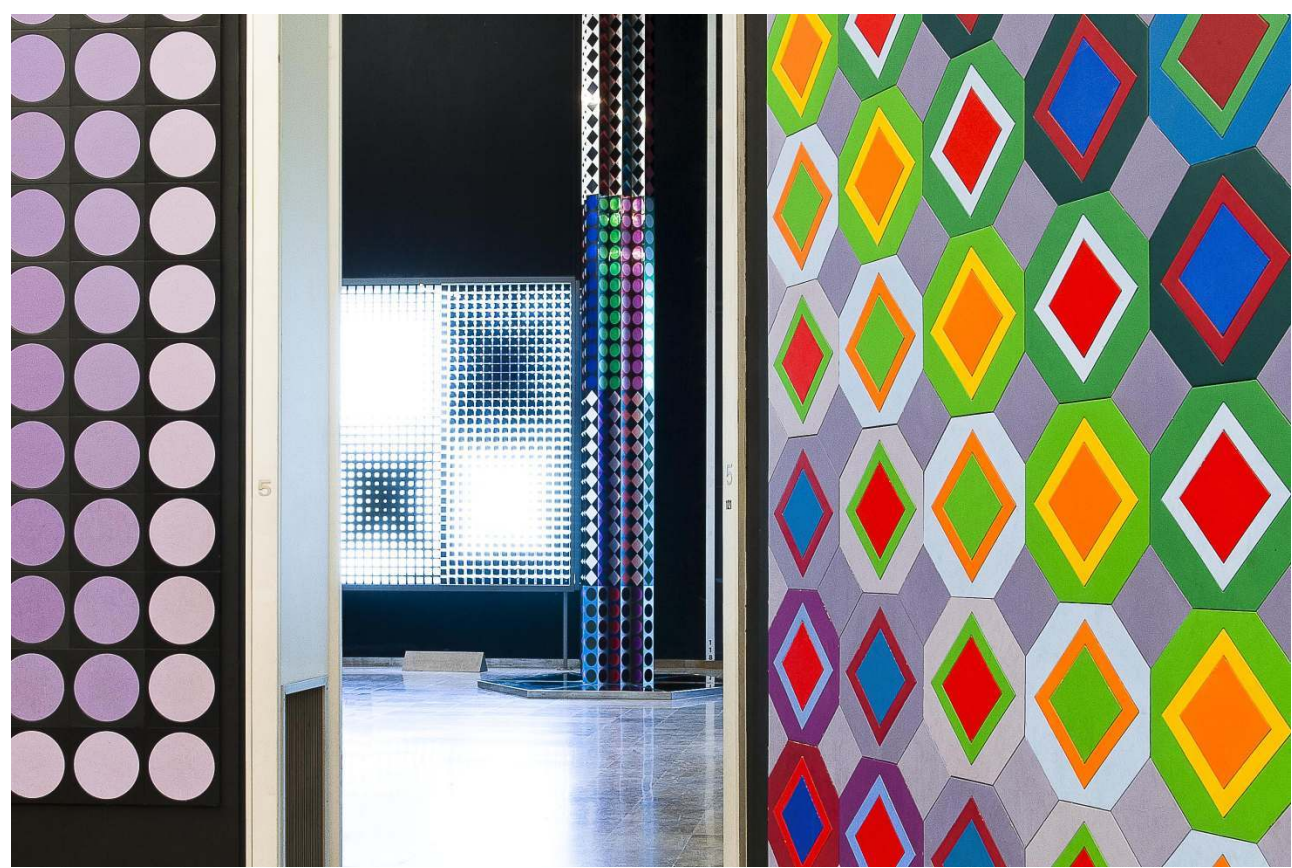

Source et copyright : Fondation Vasarely (c) Anne Fourès, Agence Luce 
Illustration 14 : Intégration Vega Anneaux, 169-1975, tapisserie réalisée à Aubusson, 528x582 cm, salle 6

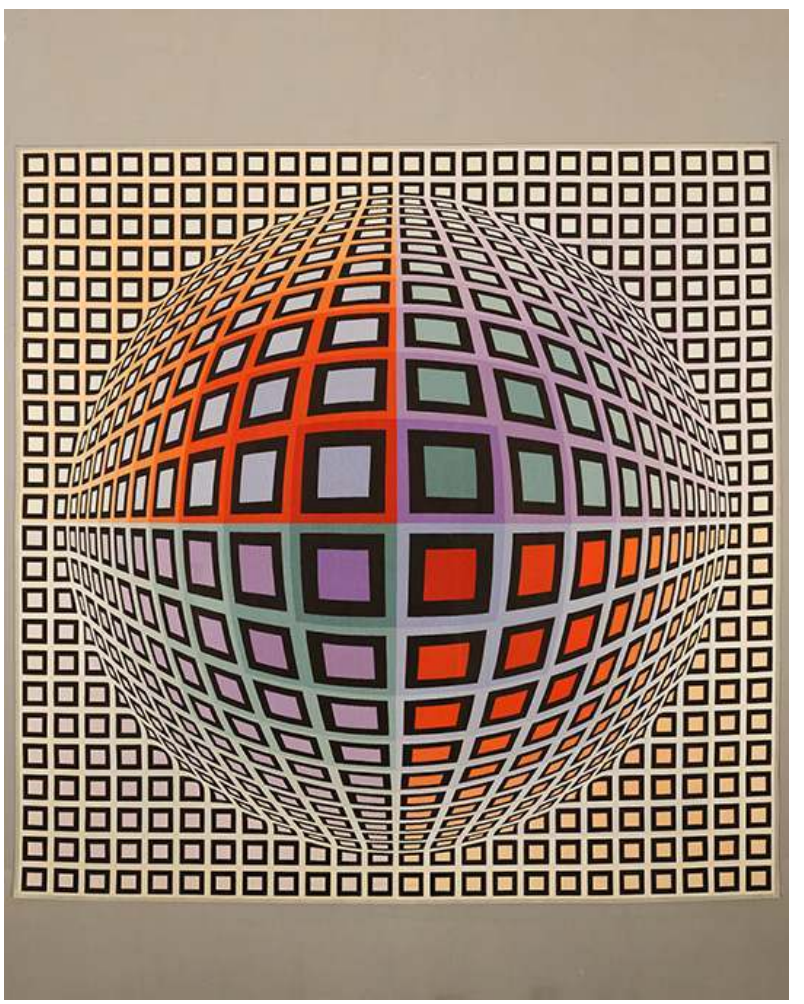

Source et copyright : Fondation Vasarely @ Anne Fourès, Agence Luce

Illustration 15 : Kroa, 1967-1969. Aluminium anodisé sur bois et moteur, 140x140x140cm, salle 4

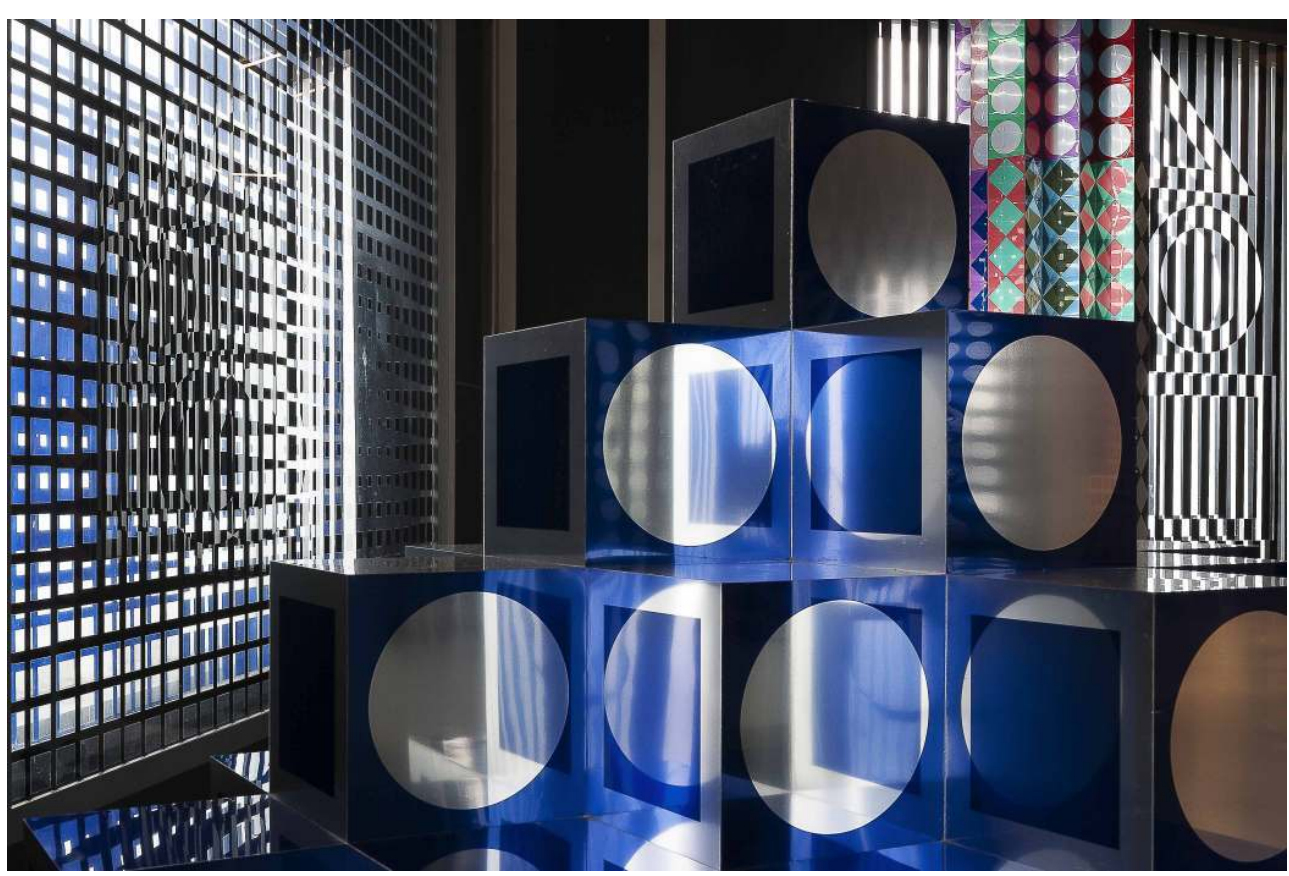

Source et copyright : Fondation Vasarely @ Anne Fourès, Agence Luce 
Avec son art cinétique, percevoir c'est être leurré, berné, dépaysé, désorienté. Vasarely ou le «bourreau de la rétine»! (Clay, 1969, p. 209). Les critiques d'art depuis 1969 sont unanimes sur le travail de Vasarely.

Son choix se porte de préférence sur des structures dont la perception ne va pas sans une certaine tension, qui frustrent l'œil en lui proposant des images visuelles à effet multiple, impossibles à saisir du premier regard. Cette incertitude, qui chez Vasarely - comme chez Josef Albers - s'intensifie parfois jusqu'à la confusion, provoque chez tous les observateurs le même effet de choc: le réflexe visuel est plus fort que la réflexion. L'accoutumance est négligeable. Notre comportement perceptuel sur lequel nous n'avons aucune prise réagit toujours comme la première fois. (Werner Spies, 1971)

Ou encore plus récemment :

Ces variations extraordinairement subtiles de formes et de couleurs jouent avec nos nerfs, et pas seulement nos nerfs optiques. Les surfaces pulsatives, les illusions particulièrement instables engagent la responsabilité du regardeur qui, contrairement à ce qui se passe devant des œuvres en effet purement décoratives (et d'autres!), ne trouve jamais vraiment sa place face au tableau (Millet, 2003)

La Fondation Vasarely à Aix représente la plus monumentale des machines à voir qu'ait réalisée Vasarely. A son ouverture, Claude Pradel-Lebar, son architecte-conseil, présageait de l'effet troublant des œuvres monumentales sur les visiteurs. "Ils percevront des mouvements immobiles, des animations inanimées, des présences qui s'effacent, des vérités qui trompent, des forces muettes qui parlent " (Claude PradelLebar, 2015, p. 60). L'édifice de Vasarely ne propose pas tant la contemplation que la confrontation. Les visiteurs seront aussi bouche bée devant l'échelle qui dépasse la géométrie (illustrations 16 et 17). Les tableaux ont la dimension des murs gris de la rue au dehors, qu'ils sont censés recouvrir. D'autres la comparent à un mausolée d'un peintre pour son œuvre (Michel, 1976, p. 22), l'étendant au caractère spirituel de l'édifice, voire religieux, le comparant à un édifice roman, avec sa porte basse à franchir, ses six chapelles hexagonales s'ordonnant autour d'une septième, le chœur (Dubrou, 1976). Ce temple du cinétisme en a marqué d'autres par son caractère ultramoderne. Cathédrale de l'an 2000, cathédrale des temps modernes (Mogui, 1976), la Fondation serait pour certains le moyen par lequel le traditionalisme provençal caractéristique d'Aix pourrait être supplanté par la civilisation post-industrielle (Eyssartel, 1976, p. 75).

Un article d'époque est particulièrement intéressant pour saisir la réception critique immédiate de cette œuvre annonciatrice de temps nouveaux. Les enfants de l'école élémentaire y seraient entrés comme dans un grand jouet, perméables à l'ambiance feérique, carnet de note et crayon en main, tandis que pour les collégiens de l'époque les œuvres auraient suscité chez eux des «pourquoi» et des «comment». Le Livre d'or apporte encore des éléments d'une réception ambivalente car fondée sur l'interprétation individuelle. "L'impression d'une agression »; "Quand on sort on est tout étourdi »; «Est-ce déjà l'an 2500? ; « Une belle synthèse du rationnel et du sensible »; "Si la Fondation était un couvent, j'entrerais dans les ordres " (Juif, 1977, p. 36). Quelques clichés de l'inauguration de la Fondation en 1976 issus des archives de la Fondation illustrent encore mieux cet état de sidération des visiteurs aux regards fermés, interrogatifs, dubitatifs, comme suspendus aux effets sibyllins de Vasarely. 
Illustration 16 : Visiteurs lors de l'inauguration de la Fondation Vasarely, 14 février 1976

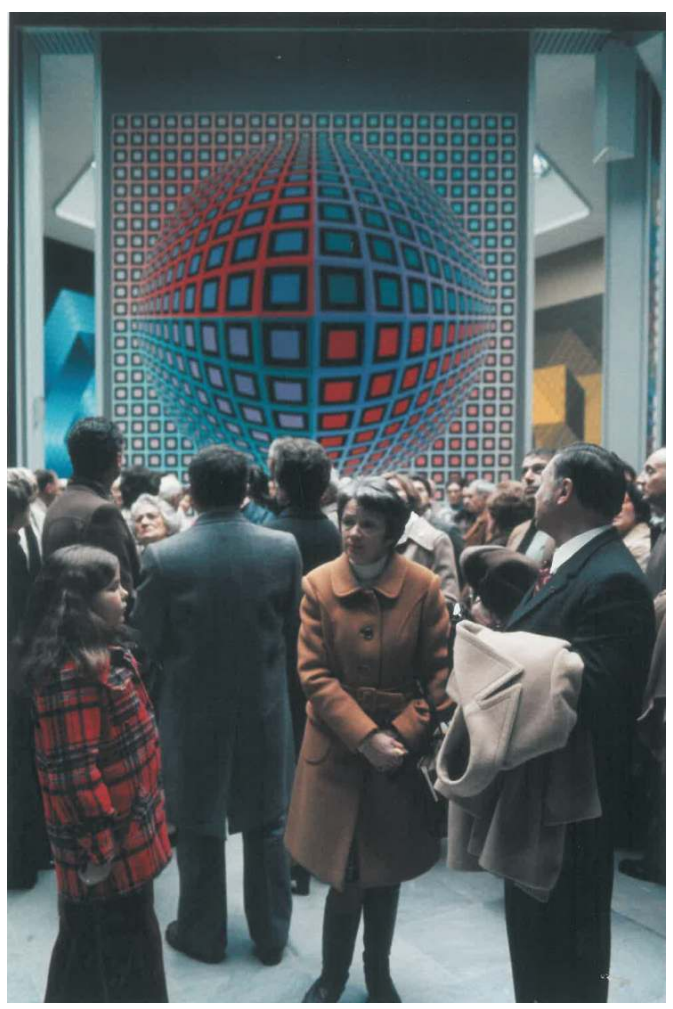

Source et copyright : Fondation Vasarely 
Illustration 17 : Visiteurs observant les œuvres monumentales lors de l'inauguration de la Fondation Vasarely, 14 février 1976

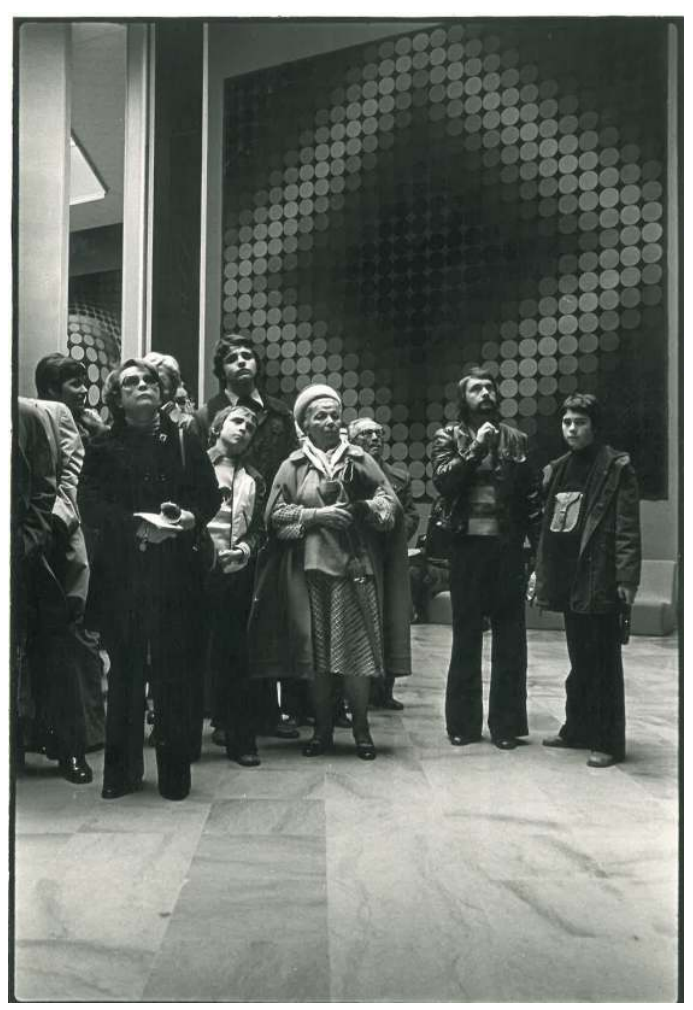

Source et copyright : Fondation Vasarely

\section{La Fondation Vasarely : une œuvre « restaurable »?}

Plus de trente ans après son inauguration, la Fondation Vasarely a vieilli. Dès 1980, des désordres importants, du reste assez mineurs, sont signalés. Des problèmes d'étanchéité sur les verrières imposent à la Fondation de prendre des mesures conservatoires, et en 1984, les quatorze coupoles de verre sont recouvertes de bâches. Les Intégrations ont été abîmées, par les infiltrations d'eau de pluie, par le contact des mains des visiteurs, par le climat intérieur déréglé par les dysfonctionnements de la climatisation. Des désordres n'ayant fait l'objet d'aucune réparation majeure ni d'entretien suivi pendant les décennies suivantes. C'est donc une Fondation presque dans son intégrité, sans stratification d'ambiances, dont hérite l'équipe pluridisciplinaire d'architectes, chargée de réaliser la réhabilitation de l'édifice ${ }^{9}$. A peine est-elle vieillotte, ternie, surannée, avec ses peintures murales qui s'écaillent, ses salissures dues aux infiltrations d'eau, son éclairage artificiel inconfortable pour pallier le manque de lumière naturelle des verrières bâchées, son mobilier aux tissus défraîchis.

\section{L'archéologie du projet au service du projet de restauration en conservation de la Fondation}

Comme Claude Pradel-Lebar, Jean Sonnier et Dominique Ronsseray en leur temps ont su traduire les desiderata de Victor Vasarely, aujourd'hui des talents complémentaires sont 
réunis autour de Pierre Vasarely pour faire revivre ce rêve réalisé de Victor Vasarely en sommeil. La complexité de l'enjeu patrimonial impose une équipe pluridisciplinaire. Une équipe d'architectes spécialistes de l'étude et de la restauration du patrimoine du $\mathrm{XX}^{\mathrm{e}}$ siècle: Jacques Repiquet, architecte mandataire, Briolle-Marro-Repiquet, architectes ; Pierre-Antoine Gatier, architecte en chef des Monuments historiques et inspecteur général des Monuments historiques, et Robert Jourdan Conservateur général du patrimoine, représentant la Conservation régionale des monuments historiques. Tous sont convaincus que la magie des lieux dans toutes ses dimensions ne peut être restaurée qu'au prix d'un long travail de recherche historique. La Conservation régionale des monuments historiques avait déjà entamé d'amples recherches sous la plume de Sylvie Denante depuis 2001, pour l'attribution du label Patrimoine du XXe siècle, puis en 2003 pour le dossier d'inscription de l'édifice sur l'inventaire supplémentaire des monuments historiques.

L'agence Pierre-Antoine Gatier ${ }^{10}$ a pris en charge une recherche substantielle centrée sur un approfondissement de la connaissance historique de la Fondation, qui a nécessité une plongée dans les archives de la Fondation et leur classement. L'étude historique, documentaire et photographique accompagnée d'un état sanitaire complet de l'édifice constitue aujourd'hui un outil de référence sur le bâtiment de la Fondation, le dossier de protection de l'édifice de 2013 s'y étant référé. L'étude a permis notamment de dévoiler la haute technicité mise en œuvre par les architectes en 1973 qui fait la spécificité de cet édifice ultramoderne. Pour la restauration, le parti d'intervention est clairement exprimé: le projet de restauration devra respecter l'intégralité de certains points notamment la composition du plan par la forme hexagonale prédominante, de la façade et des verrières zénithales de la couverture, ainsi que la polychromie et la conception paysagère, pour conserver ou restituer les dispositions et ambiances intérieures (Gatier, 2011, p. 45).

L'approche patrimoniale des architectes dans cette opération de restauration en conservation est clairement scientifique et technique. Elle consiste pour l'essentiel à concilier les exigences de restauration à l'identique et la conformité aux contraintes techniques actuelles. L'esprit de grande modestie, d'innovation technique et d'expérimentation des architectes de 1976 doit être repris par les architectes contemporains. La compatibilité des matériaux mis en œuvre originellement avec les usages et les réglementations actuels doit être visée. Mais la mise à niveau technique impose dans certains cas, une adaptation des dispositions originelles. La restauration de l'enveloppe et de ses panneaux œuvres, du mur-rideau, des verrières et la réfection de l'équipement technique et en particulier le chauffage, la ventilation, la climatisation sont indispensables pour rétablir le confort d'ambiance nécessaire à la visite et aux conditions de conservation préventive des œuvres.

\section{Les verrières zénithales : priorité à l'authenticité, à la technicité ou à l'ambiance?}

31 L'élément qui, en son temps, a le plus contribué à l'allure extérieure et à l'ambiance limpide intérieure de la Fondation est celui qui aujourd'hui pose le plus de difficulté en termes d'éthique de restauration en conservation: les verrières zénithales (illustration 18). Restaurer l'ambiance lumineuse permettra de se rapprocher de l'expérience originale des espaces. 
Protégées des intempéries depuis 1984 par des bâches semi-occultantes, les verrières de toiture devaient retrouver leur rôle d'origine. Le projet initial de restauration prévoyait l'ajout de sur-verrières de protection au-dessus des verrières d'origine qui devaient être restaurées à l'identique. L'occultation aurait alors été possible par l'ajout de stores triangulaires enroulables pour certains espaces de travail. Cette première solution a dû être abandonnée pour son coût trop élevé, mais aussi à cause de la surcharge des surverrières qui auraient imposé une poutraison supplémentaire en toiture alors même que la solidité de la charpente était confirmée. Les sondages effectués ont confirmé que la restauration des verrières et de la charpente nécessite un démontage de l'ensemble des châssis vitrés. La solution avancée par le conservateur régional des Monuments historiques consiste à conserver les verres d'origine et à appliquer un film adhésif sur le vitrage extérieur conservé pour filtrer la lumière pour la conservation préventive des œuvres. Aujourd'hui encore, ce débat n'est pas tranché. Puisque les châssis vitrés doivent être tous démontés pour leur nettoyage, les architectes proposent que le verre supérieur d'origine soit remplacé par un verre performant sur lesquels des châssis de protection solaire amovibles peuvent être rapportés. Le vitrage d'origine serait conservé sur plusieurs verrières " témoins ", abritant les œuvres les moins sensibles à la lumière.

La question épineuse de la restauration des verrières repose sur des aspects à la fois théoriques de conservation, techniques de mise en œuvre et esthétiques d'ambiances. Il semble que les possibilités techniques l'emportent sur la volonté de sauvegarde des éléments d'origine. Sur certaines alvéoles, la priorité est donnée aux exigences muséographiques de conservation des œuvres, vient ensuite le confort de visite, et non la restitution du confort de visite initial. Les ambiances sont dans ce cas figées au rang de conséquence des décisions prises en terme de techniques de conservation. L'idée des architectes pour la restauration des verrières est pourtant bien de ne pas oublier les intentions initiales de Vasarely qui consistaient à être dedans comme si on était dehors.

Illustration 18 : Restauration de la verrière de la salle 4, décembre 2014

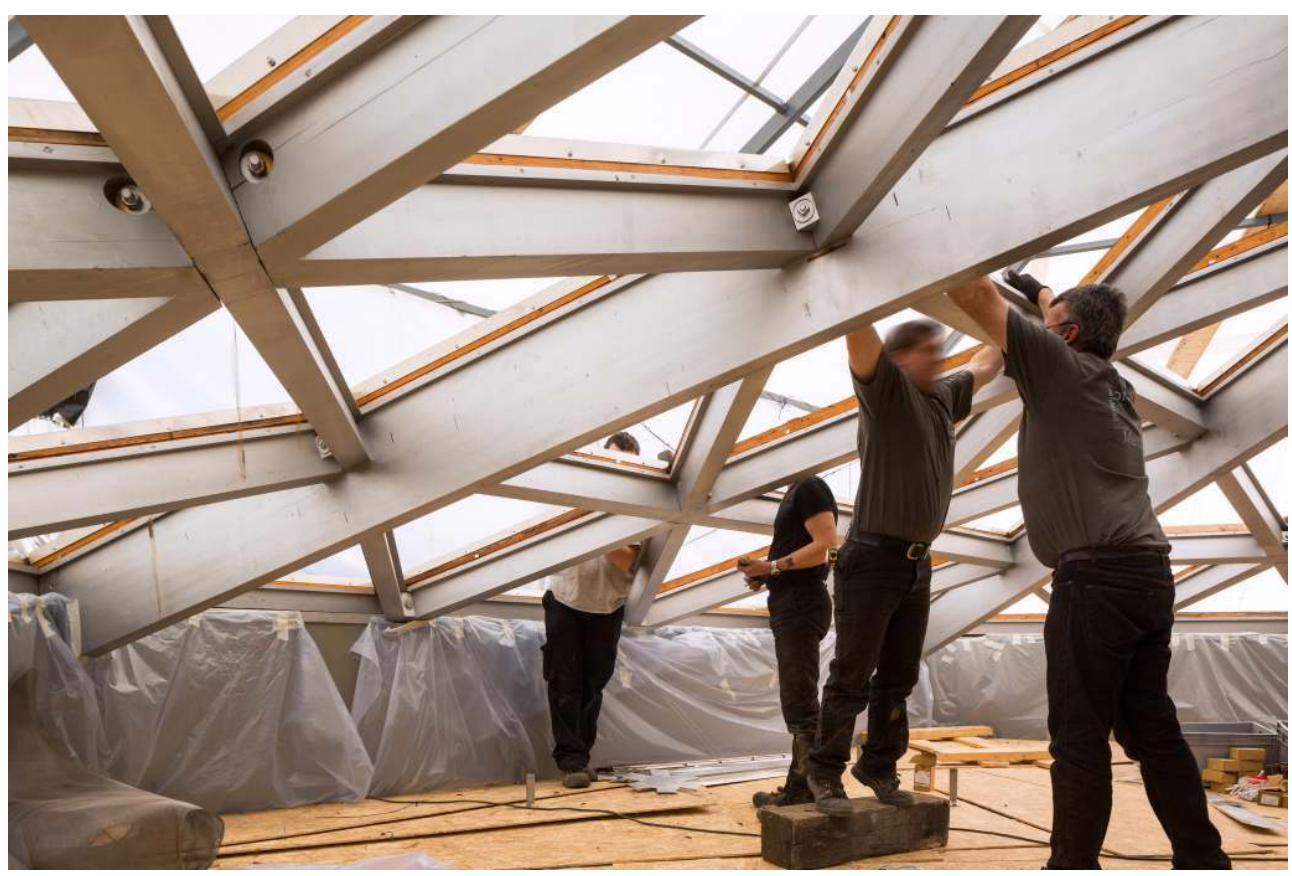

Source et copyright : Fondation Vasarely (c) Gabrielle Voinot 


\section{La façade-œuvre ou comment restaurer une robe Courrèges sans pouvoir la découdre}

34 n'a été atteinte qu'au prix d'une recherche et d'une mise en œuvre expérimentales ${ }^{11}$. Les $3000 \mathrm{~m}^{2}$ de surface de façade ne devaient se lire que comme une seule et même œuvre monumentale et non comme un assemblage de 200 pièces de tôle noir et blanc. Mais jamais le lavage annuel préconisé initialement n'a été réalisé. Dégradations, salissures ont eu raison de l'effet original: les joints se perçoivent, l'assemblage est démasqué, l'œil n'est plus trompé. Le projet de restauration prévoyait le démontage de chacune des plaques permettant ainsi leur restauration aisée mais aussi l'incorporation d'une importante isolation thermique. Mais un premier essai en mars 2016 a dévoilé l'impossibilité de démontage. C'est qu'en 1974 la réalisation de cette façade-prototype avait fait appel autant aux techniques les plus industrialisées qu'à l'artisanat le plus ingénieux. Une peau réalisée sur-mesure, qui est à l'architecture ce que la haute couture est à la mode. Si les plans préconisaient un système d'assemblage industriel et mécanique démontable, les difficultés d'exécution en 1974 ont introduit l'ajout de multiples pièces de fixation complémentaires pour rendre l'ouvrage possible, produisant une œuvre indémontable. Il s'agit bien de redonner de l'éclat à une robe Courrèges sans pouvoir la découdre...

La mise en œuvre expérimentale originale impose aux architectes aujourd'hui de renouveler une posture expérimentale qui se fonde sur des tests, des essais, des prototypes. Comme Sylvie Denante l'a avancé, la difficulté réside dans le fait que «la Fondation Vasarely n'est pas une architecture d'architecte, mais une architecture de communication » (Sylvie Denante, 2012, p. 4). Pour que l'œuvre monumentale de la façade persiste à faire signe, pour qu'elle continue de flouer la perception du visiteur, les architectes doivent « restaurer en innovation ».

\section{Restaurer une ambiance ou comment retrouver l'expérience initiale de l'espace}

La patrimonialisation de la Fondation et sa restauration en conservation tendent à figer l'état matériel de l'édifice aux intentions initiales de son auteur, et par extension elles figent aussi les atmosphères si emblématiques de l'univers de Vasarely, les transformant en ambiances patrimoniales. La restauration en conservation a le mérite de transmettre aux générations futures un témoignage unique de son temps, une œuvre avant-gardiste qui continue d'avoir une place singulière dans le développement de l'histoire. La recherche d'une vérité d'ambiance passée reste pourtant un leurre (Simonnot, 2012), peut-être aussi important que celui que Vasarely nous inflige dans l'observation de ses œuvres cinétiques. La caractérisation d'une ambiance ne dépend pas seulement de la restitution de verrières, de la remise en état de peintures murales ou de la restauration des façades en aluminium. Les facteurs spatiaux et physiques ne suffisent pas à déterminer une ambiance. L'ambiance se qualifie aussi et surtout par l'individu qui le pratique, un individu tributaire de son milieu social, culturel et en particulier de son époque (Belakehal, 2012). Il n'y a qu'à jeter à nouveau un coup d'œil à ces clichés de 1976 lors de l'inauguration. Les visages des visiteurs en disaient long sur leur choc émotionnel, 
sur leur saisissement, leur incrédulité face à ces œuvres monumentales nouvelles (illustration 19). En quarante ans l'art contemporain - avec ses divers courants comme le land art, les graffiti, les arts à composante électronique,... - a distancé l'art optique. Au surplus, la reconnaissance patrimoniale de l'édifice de la Fondation est concomitante avec un regain d'intérêt pour l'art optique et cinétique, après une longue période d'indifférence. En 2005, l'exposition strasbourgeoise, "L'œil moteur: art optique et cinétique, 1950-1975 ", instaure une relecture critique de ce mouvement expérimental, interactif, issu de l'émergence d'une société technique. En 2013, quatre événements, à la tête desquels se place l'exposition du Grand Palais, « Dynamo : un siècle de lumière et de mouvement dans l'art, 1913-2013 ", permettent d'inscrire le mouvement de l'art optique dans une perspective historique ${ }^{12}$. Mais le temps a passé aussi sur cet art. Le futur, rêvé par Vasarely, n'a pas eu lieu. L'optimisme de ses œuvres accessibles à des publics populaires, appréhendables par les seules facultés normales de perception du spectateur, reproductibles à grande échelle dans l'espace urbain, est passé. L'histoire nous a montré ces œuvres ludiques tels des événements plastiques, dont la signification artistique comptait moins que la communication artistique. Vulgarisés sous différents supports, popularisés, ces œuvres ont fait l'objet d'un processus de consommation, qui aujourd'hui les éloigne de l'effet d'étonnement, d'incrédulité originel. Le visiteur du XXI ${ }^{\mathrm{e}}$ siècle n'est plus le même que celui de l'inauguration de la Fondation en 1976.

Illustration 19 : Visiteurs interpellés par les œuvres lors de l'inauguration de la Fondation Vasarely, 14 février 1976

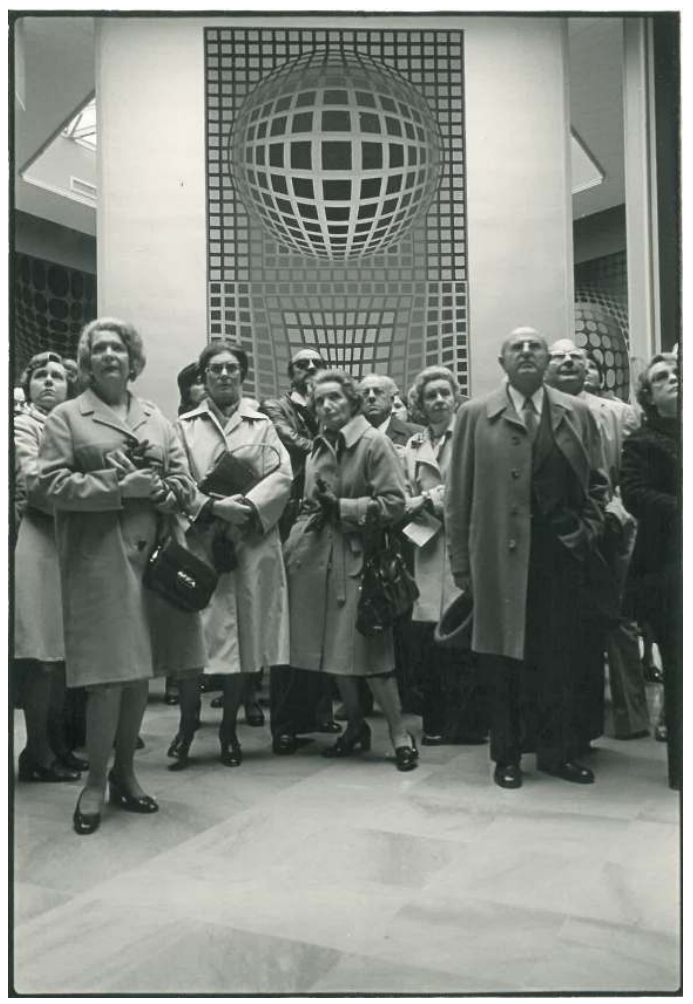

Source et copyright : Fondation Vasarely

Mais en figeant l'ambiance de la Fondation dans les années 1970, la restauration en conservation des œuvres et des espaces a le mérite de montrer combien l'art de Vasarely était prophétique. Que l'on ne s'y trompe pas; ses œuvres, qui semblent avoir été 
réalisées par ordinateur, datent bien de la fin des années 1970, lorsque l'informatique n'en était qu'à ses balbutiements. Dans sa recherche d'un langage universel et compréhensible par tous, Vasarely a élaboré un alphabet de formes et de couleurs. Convaincu que les mathématiques détenaient une beauté plastique, il en a recueilli toute la puissance combinatoire et permutative. En remplaçant les couleurs par des chiffres, il a encodé ses images sans ordinateur. Cette codification des éléments de création plastique l'autorise à produire un art programmable, reproductible. Bien avant l'avènement de la nouvelle révolution technologique, Vasarely avait saisi le potentiel de la programmation en ce qu'elle permettra non "plus de faire mais de savoir faire faire ». A l'heure où le numérique triomphe, l'œuvre de Vasarely apparaît d'autant plus visionnaire.

38 Avec la recherche d'authenticité du maximum de matière, du caractère avant-gardiste des œuvres mais aussi des expériences spatiales, la restauration de la Fondation inscrit toute l'œuvre dans sa dimension à la fois prémonitoire (avoir fait le lien entre art et informatique) et surannée, en somme dans toute son actualité. C'est pour cette raison que la restauration en conservation ne serait complète sans deux points majeurs.

Le premier consiste en la restauration des Présentoirs, système ingénieux imaginé par Vasarely pour dévoiler au public ses 798 dessins prévus à une échelle monumentale pour embellir la cité (illustration 20). Présentés dans un format réduit de $84 \times 78 \mathrm{~cm}$, ces documents étaient répartis dans 22 présentoirs. L'intérêt était de montrer le maximum d'œuvres en utilisant le minimum d'espace. Une présentation en cimaise traditionnelle aurait nécessité dix grandes salles de $25 \times 25 \mathrm{~m}$ chacune. Vasarely propose que le visiteur, confortablement installé sur une banquette, actionne lui-même le mécanisme du présentoir (aujourd'hui altéré) faisant défiler les œuvres à la vitesse de 30 secondes par image. Ce dispositif innovant est un maillon indissociable pour saisir son désir d'intégrer l'art à la vie quotidienne. La reconnaissance de ces Présentoirs par le biais d'une inscription au même titre que l'ensemble de la Fondation permettrait de parfaire la restauration en conservation de la Fondation, lui rendant son statut de lieu entièrement tourné vers l'avenir dans les années 1970, prophétique de l'ère numérique. 
Illustration 20 : Les Présentoirs dans leur disposition d'origine, dans les alvéoles 10 et 11 à l'étage

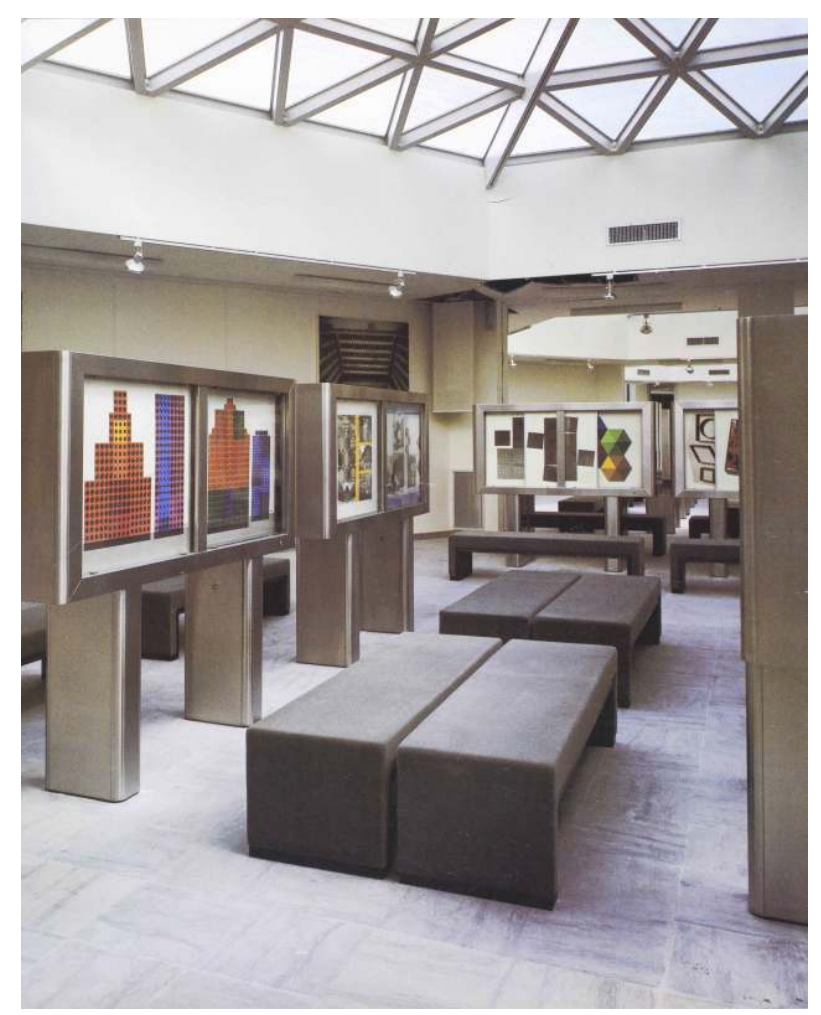

Source et copyright : Fondation Vasarely @ $\subset$ Anne Fourès, Agence Luce

Le deuxième point est celui du projet d'une future extension de la Fondation qui a été confiée aux mêmes architectes en charge de la restauration, associés à l'architecte Marc Barani. La Fondation et sa restauration ne devraient pas faire oublier le but supérieur que Vasarely voulait atteindre à travers elle. L'action de la Fondation devait avant tout être sociale, tournée vers les problèmes d'intégration d'éléments plastiques dans l'architecture et l'urbanisme. L'enjeu était de "promouvoir une forme d'art véritablement démocratique, ouvrir enfin la voie à une vision architectonique moderne de l'environnement humain d'aujourd'hui et de demain " (Vasarely, 1979a, p. 8). Mais malheureusement, les projets concrets n'ont pas suivi. La Fondation Vasarely n'est restée qu'une démonstration intime, un coup d'essai confidentiel. Elle n'est restée qu'une projection d'ambiance gaie sur la ville grise. Car la Fondation, malgré ses statuts de centre de recherches ouvert sur la ville, reste encore, par sa configuration spatiale d'édifice fermé sur lui-même, centrée sur l'œuvre de son auteur. C'est cette ambiance d'un espace spirituel que restituent actuellement avec justesse les architectes en charge de la restauration en conservation. Elle a le grand mérite de faire de la Fondation un grand témoignage intact de l'utopie "vasarélienne ». Elle permet aux visiteurs d'aujourd'hui, munis d'une épaisseur culturelle, de mesurer ce que la démocratisation de l'art avait de bon, comme de comprendre tout ce que son projet esthétique pour la société future avait d'illusoire. A charge peut être pour les architectes de la future galerie d'exposition de $1000 \mathrm{~m}^{2}$ de créer ce Centre de création polymorphe auquel tenait tant le plasticien, un lieu de création contemporaine tendu vers l'avenir et ouvert sur la ville, mais toujours relié à cette architecture-manifeste pleinement respectée dans son authenticité qui fait partie de notre patrimoine. 
Envisager l'insertion de l'ambiance dans une démarche patrimoniale ne serait alors pas simplement « rendre actuelles les ambiances surannées » d'un musée, mais plutôt aller à la découverte des expériences sensibles imaginées autrefois et les réinstaller à travers des dispositifs capables de générer aujourd'hui les sensations pensées hier, à travers des entités qui réhabiliteraient les dimensions immatérielles avec des pratiques actuelles revisitées et contemporaines, dans une sorte de stratégie d'amplification de l'ambiance imaginable.

\section{BIBLIOGRAPHIE}

Ameline, Jean-Paul (eds.). 2013. Victor Vasarely. De l'œuvre peint à l'œuvre architecturé. Catalogue de l'exposition éponyme. Paris : Editions Hermann.

Amphoux, Pascal, (eds.). 1998. La notion d'ambiance. Une mutation de la pensée urbaine et de la pratique architecturale. Paris : Editions du PUCA.

Augoyard, Jean-François. 1998. Eléments pour une théorie des ambiances architecturales et urbaines. In : Les Cahiers de la Recherche Architecturale, $\mathrm{n}^{\circ}$ 42/43, $3^{\mathrm{e}}$ trimestre, p. 13-23.

Belakehal, Azeddine. 2012. Ambiances patrimoniales. Problèmes et méthodes. In : Thibaud, JeanPaul ; Siret, Daniel (eds.). 2012. Ambiances in action-Ambiances en acte(s). Proceedings of the 2nd International Congress on Ambiances - Actes du $2^{\text {nd }}$ Congrès International sur les Ambiances, Montréal, 19 - 22 septembre 2012. Grenoble : Réseau international Ambiances : Ecole nationale supérieure d'architecture de Grenoble, p. 505-510.

Bokern, Anneke. 2008. Polychromie und Glück. Die Fondation Vasarely in Aix-en-Provence, 1973-1976. In : Werk, bauen + wohnen, n 7-8, p. 22-29.

Brandi, Cesare. 2011. Théorie de la restauration. Paris : Éditions Allia. [1963. Teoria del restauro. Roma : Edizioni di Storia e Letteratura].

Clay, Jean. 1969. Victor Vasarely. In : Visages de l'art moderne. Lausanne : Rencontres, p. 209.

Dahhan, Bernard. 1979. Victor Vasarely ou la Connaissance d'un art moléculaire : la nouvelle plastique et les sciences modernes. Paris : Denoël, Gonthier.

Demoriane, Hélène. 1976. Vasarely : la maison du prophète. In : Le Point, $\mathrm{n}^{\circ}$ 177, 9 février, p. 87-89.

Denante, Sylvie. 2003. Fondation Vasarely. Dossier d'inscription de la Direction Régionale des Affaires Culturelles. DRAC PACA.

Denante, Sylvie. 2012. Fondation Vasarely. Dossier de protection de la Direction Régionale des Affaires Culturelles. DRAC PACA.

Diehl, Gaston. 1973. Vasarely. Paris : Flammarion.

Dubrou, Robert. 1976. L'art en mutation à la Fondation Vasarely. In : Options, $\mathrm{n}^{\circ}$ 105, mars, p. 50-53.

Eyssartel, Jean-Louis. 1976. Avant-scène pour Vasarely. In : Arts et Spectacles, p. 74-75.

Fondation Vasarely. 1988. Aix-en-Provence : Editions Fondation Vasarely. 
Gatier, Pierre-Antoine. 2011. Réhabilitation de la Fondation Vasarely, étude diagnostic. Architecte en chef des Monuments historiques. Tapuscrit.

Graf, Franz et Delemontey, Yvan (eds). 2012. Architecture industrialisée et préfabriquée : connaissance et sauvegarde. Lausanne : Presses polytechniques et universitaires romandes.

Juif, Paul. 1977. La Fondation Vasarely. In : L'Education, n 313, 24 mars, p. 32-36.

Michel, Jacques. 1976. Le mausolée d'un peintre pour son œuvre. In : Le Monde, 17 février, $n^{\circ} 9664$, p. 22.

Millet, Catherine. 2003. Victor Vasarely. In: Art Press, $n^{\circ}$ 288, avril, n.p.

Mogui, Jean-Pierre. 1976. Vasarely ou l'art de la façade. In : Le Figaro, 14-15 février, n.p.

Mouillefarine, Laurence. La deuxième vie de Vasarely. Spéciale Biennale. In : $A D$, sptembreoctobre 2010, p. 7-10.

Pradel-Lebar Claude ; Gatier, Pierre-Antoine ; Repiquet, Jacques ; Vasarely, Pierre (et alii). 2015. La Fondation Vasarely. Victor Vasarely, visionnaire utopiste. Paris : Beaux-Arts Editions, TTM Editions.

Repiquet, Jacques. 2016. Restaurer la Fondation Vasarely. In : Patrimoine(s) en Provence-Alpes-Côted'Azur, La Lettre d'information de la DRAC, n³1, mars 2016, en ligne.

Rosille, Frédéric. 2007. Musicalité de l'œuvre plastique de Victor Vasarely. In : Le carré bleu, n² 2.

Simonnot, Nathalie. 2012. Le paradoxe de la patrimonialisation des ambiances. In : Thibaud, JeanPaul ; Siret, Daniel (eds.). 2012. Ambiances in action - Ambiances en acte(s). Proceedings of the 2nd International Congress on Ambiances - Actes du $2^{\text {nd }}$ Congrès International sur les Ambiances, Montréal, 19 - 22 septembre 2012. Grenoble : Réseau international Ambiances : Ecole nationale supérieure d'architecture de Grenoble, p. 33-38.

Sonnier, Jean. (s.d.). Fondation Vasarely, Aix-en-Provence. Tapuscrit, 32 feuillets. Archives de la Fondation Vasarely.

Spies, Werner. 1971. Victor Vasarely. Paris : Editions Cercle d'Art.

Toulier, Bernard. 2000. Architecture et patrimoine du XXe siècle en France. Paris : Editions du Patrimoine.

Vasarely, Pierre ; Pradel-Lebar, Claude ; M. Cunnigham, John ; et alii. 2010. La Fondation Vasarely, de l'op art à la cité polychrome du bonheur. Aix-en-Provence : Images en manœuvre éditions.

Vasarely, Victor. 1970. Plasti-cité, l'oeuvre plastique dans votre vie quotidienne. Paris : Castermann.

Vasarely, Victor. 1972. Notes brutes. Paris : Denoel/Gonthier.

Vasarely, Victor. 1979. Vasarely plasticien. Paris : Laffont.

Vasarely IV. 1979. Neuchâtel : Editions du Griffon.

\section{NOTES}

1. Depuis le décès de Claire Vasarely, épouse de Victor Vasarely, en 1990, la Fondation Vasarely a connu des vicissitudes (pillage de l'œuvre de Vasarely, perte des fonds et des collections, arbitrage successoral, etc.) qui ont fait l'objet de procédures judiciaires dont certaines sont encore en cours, consultables sur le site internet de la Fondation (rubrique "Procédures »). En 2015, la cours de Cassation a reconnu Pierre Vasarely, petit-fils du peintre, titulaire du droit moral et seul légataire universel du plasticien. 
2. Goven, François. 2013. Le bâtiment de la Fondation Vasarely, un monument historique ? In : Ameline, Jean-Paul (eds.). Catalogue d'exposition De l'oeuvre peint à l'œuvre architecturé. p. 97-98.

3. Son œuvre plastique peut être découpée par périodes. 1929-1946, période graphique : au cours de cette première période, Vasarely crée un alphabet plastique basé sur des combinaisons infinies ; 1947-1958, période Belle-Isle : sa démarche abstraite se concrétise ; 1951-1958, période Denfert ; 1948-1958, période Cristal-Gordes ; 1948-1960, périodes pendant lesquelles il travaille sur le thème du mouvement (travail qu'il expose en 1955 à la galerie Denise René à Paris) et publie son Manifeste Jaune qui annonce la notion de «plastique cinétique » principe selon lequel le mouvement relève de la perception du regard et période Noir-Blanc ; 1960-1964, le folklore planétaire. Pour une meilleure compréhension des œuvres plastiques de Vasarely, se référer aux analyses de Pauline Mari, Claudine Ripoll, Jean-Pierre Ameline, Pierre Vasarely.

4. Le réseau des "musées Vasarely» est complété par le musée Vasarely de Pécs en Hongrie, maison natale de l'artiste, inauguré en juin 1976 ; le «Vasarely Center » inauguré à New York en mai 1978, qui devait être le double de la Fondation Vasarely d'Aix, et fermé depuis la fin des années 80 ; le Centre Vasarely à Oslo en Norvège ouvert en 1982 et fermé en 1987 ; le Musée Vasarely au Château Zichy à Budapest inauguré en 1987. Ce réseau visait à populariser l'art et le rendre accessible au plus grand nombre.

5. La première citation est issue de Vasarely, plasticien. Un homme et son métier, p. 214 ; la seconde d'une note rédigée par Victor Vasarely et citée par Pierre Vasarely sur le site internet de la Fondation Vasarely.

6. Victor Vasarely avait déjà collaboré avec Jean Sonnier. Dans le cadre de la restauration et de la transformation du château de Gordes en musée didactique, l'architecte en chef des Monuments historiques avait installé les collections Vasarely dans l'édifice, classé monument historique depuis 1931.

7. Arpád Feszty (1856-1914), L'arrivée des Hongrois, cyclorama gigantesque représentant la conquête magyare de la Hongrie en 896.

8. Exposition "Responsive Eye" au Musée d'Art Moderne de New York en 1965 consacrée à l'Art optique à laquelle participe Victor Vasarely.

9. L'équipe de maîtrise d'œuvre chargée de la réhabilitation et de l'extension de la Fondation Vasarely à Aix-en-Provence est composée des architectes suivants : Jacques Repiquet, Mandataire - Briolle-Marro-Repiquet Architectes, Pierre-Antoine Gatier, Architecte en chef des Monuments historiques, Inspecteur Général des Monuments Historiques et Marc Barani, qui a plus particulièrement étudié le projet d'extension.

L'équipe est complétée par Birgitte Fryland "Sceno ", Scénographe / Philippe Deliau " ALEP », paysagiste / Robert-Jan Van Santen «VS-A » ingénierie de l'enveloppe, spécialistes de la restauration du verre et du métal / Kléber Daudin ingénierie thermique et fluides / AUXITEC ingénierie structure et économie / Adrien Amantini OPC / APAVE Roberto Sadrin CT.

10. Le dépouillement des archives a été élaboré par les architectes-collaborateurs de l'agence Pierre-Antoine Gatier : Fanny Houmeau-Schmitt et Gwendoline Thepaut, l'historienne Audrey De Cillia, ainsi qu'un stagiaire de la Fondation Jean Saint-Pierre.

11. Cf. dossier sous la direction de Jacques Repiquet, "Restaurer la Fondation Vasarely", Patrimoine(s) en Provence-Alpes-Côte-d'Azur, La Lettre d'information de la DRAC, n³1, mars 2016. Disponible sur: http://www.infos-patrimoinespaca.org/index.php? menu=9\&num_article=382\&mp=20\&cptcom=0\&dos=33 (consulté le 28 juillet 2016).

12. En 2013: exposition sur le Groupe de recherche d'art visuel (GRAV), Mouvement lumière participation. GRAV 1960-1968 au Musée des Beaux-Arts de Rennes, rétrospective de Julio Le Parc au Palais de Tokyo et de Soto au Centre Pompidou, Paris. 


\section{RÉSUMÉS}

En 1973, le plasticien Victor Vasarely se fait architecte en construisant une Fondation, un centre architectonique, qui portera son nom, à Aix-en-Provence. Cette architecture singulière, manifeste de l'art optique, interpelle par son enveloppe autant que par ses intérieurs: à l'extérieur, un monument-écrin en noir et blanc versus à l'intérieur, une explosion de couleurs grâce aux 42 œuvres monumentales, les Intégrations de Vasarely. Quarante ans après son édification, le bâtiment a vieilli mais son état d'authenticité est restaurable. Une opération de restauration en conservation est entreprise depuis 2011. Et l'édifice a été inscrit (2003), puis classé Monument historique (2013). La patrimonialisation de cette œuvre emblématique des Trente Glorieuses questionne. S'agit-il de restaurer les qualités matérielles intérieures et extérieures de l'édifice ou avant tout l'esprit du lieu? Pour un édifice dont la particularité est d'être une machine à illusions, est-il possible de recomposer les conduites perceptives et comportementales des visiteurs à l'identique? Questionner la dimension sensorielle de la restauration-conservation de la Fondation revient ici à sonder la notion ambiguë d'« ambiance patrimoniale».

In 1973, the artist Victor Vasarely turned architect by building the Foundation, an architectonic centre which was to bear his name, in Aix-en-Provence. This singular architectural manifestation of optical art challenges us by its envelope as much as by its interior: a monument in black and white on the outside versus an explosion of colour, thanks to 42 monumental works, Integrations by Vasarely. Forty years after its construction, the building has aged, but its authenticity can be restored. Conservation and restoration measures have been under way since 2011. The building was listed in 2003, then classified as a historic building in 2013. Making this emblematic work of Les Trente Glorieuses, the thirty "glorious" years between 1945 and 1975, part of our heritage is questioned. Is it a question of restoring the building's internal and external material qualities, or is it above all the spirit of the place that counts? For a building whose characteristic is to be a machine with illusions, is it possible to reproduce visitors' perceptual and behavioural responses identically? To question the sensory dimension of the restoration / conservation of the Foundation amounts here to probing the ambiguous concept of "heritage ambiance".

\section{INDEX}

Keywords : optical art, Victor Vasarely, architecture of the Thirty Glorious Years, environment, conservation/restoration, perception

Mots-clés : art optique, Victor Vasarely, architecture des Trente Glorieuses, ambiance, restauration en conservation, perception 


\section{AUTEUR}

\section{ANA BELA DE ARAUJO}

Architecte de formation, docteur en histoire de l'art (Université de Paris I, Panthéon-Sorbonne), Ana Bela de Araujo est maître-assistante à l'École nationale supérieure d'architecture de Marseille. Chercheur au LACTH (laboratoire de recherche de l'ENSA de Lille) et chercheurassociée à INAMA (laboratoire de recherche de l'ENSA de Marseille), elle a mené différents travaux de recherches sur l'histoire de l'architecture du XXe siècle. Elle a publié des articles dans des revues d'art et d'architecture et dans des catalogues d'exposition ainsi qu'un ouvrage intitulé Marie Dormoy / Auguste Perret. Correspondance. 1922-1953 (Éditions du Linteau, 2009).

Contact : dearaujo.a@wanadoo.fr 